\title{
Antifungal Microbial Agents for Food Biopreservation-A Review
}

\author{
Marcia Leyva Salas ${ }^{1,2}$, Jérôme Mounier ${ }^{1}$, Florence Valence ${ }^{2}$ (D) , Monika Coton ${ }^{1}$, Anne Thierry ${ }^{2}$ \\ and Emmanuel Coton 1 ,* \\ 1 Laboratoire Universitaire de Biodiversité et Ecologie Microbienne (LUBEM EA3882), Université de Brest, \\ Technopole Brest-Iroise, 29280 Plouzané, France; marcia.leyvasalas@univ-brest.fr (M.L.S.); \\ jerome.mounier@univ-brest.fr (J.M.); monika.coton@univ-brest.fr (M.C.) \\ 2 UMR1253 Science et Technologie du Lait et de l'Euf, INRA, Agrocampus Ouest, 65 rue de Saint Brieuc, \\ 35000 Rennes, France; florence.valence-bertel@inra.fr (F.V.); anne.thierry@inra.fr (A.T.) \\ * Correspondence: emmanuel.coton@univ-brest.fr; Tel.: +33-2-9091-5111; Fax: +33-2-9091-5100
}

Academic Editors: Régine Talon and Monique Zagorec

Received: 9 May 2017; Accepted: 24 June 2017; Published: 8 July 2017

\begin{abstract}
Food spoilage is a major issue for the food industry, leading to food waste, substantial economic losses for manufacturers and consumers, and a negative impact on brand names. Among causes, fungal contamination can be encountered at various stages of the food chain (e.g., post-harvest, during processing or storage). Fungal development leads to food sensory defects varying from visual deterioration to noticeable odor, flavor, or texture changes but can also have negative health impacts via mycotoxin production by some molds. In order to avoid microbial spoilage and thus extend product shelf life, different treatments-including fungicides and chemical preservatives-are used. In parallel, public authorities encourage the food industry to limit the use of these chemical compounds and develop natural methods for food preservation. This is accompanied by a strong societal demand for 'clean label' food products, as consumers are looking for more natural, less severely processed and safer products. In this context, microbial agents corresponding to bioprotective cultures, fermentates, culture-free supernatant or purified molecules, exhibiting antifungal activities represent a growing interest as an alternative to chemical preservation. This review presents the main fungal spoilers encountered in food products, the antifungal microorganisms tested for food bioprotection, and their mechanisms of action. A focus is made in particular on the recent in situ studies and the constraints associated with the use of antifungal microbial agents for food biopreservation.
\end{abstract}

Keywords: antifungal; bioprotection; biocontrol; food; post-harvest; fungi; lactic acid bacteria; propionibacteria; Bacillus; fermentates; molecules

\section{Introduction}

Today, food losses are a major concern worldwide especially with an ever-growing world population and the fact that approximately one-third of all food produced for human consumption is either lost or wasted [1]. Each year, an estimated 1.3 billion tons of food are lost or wasted as stated by the Food and Agriculture Organization of the United Nations [2]. When considering each food category, these losses correspond to $40-50 \%$ of root crops, fruits, and vegetables; $35 \%$ of fish and seafood; $30 \%$ of cereals; and $20 \%$ of meat, oil seed, and dairy products [2]. In developing countries, post-harvest loss rates are high with $30-40 \%$ occurring during the post-harvest and processing stage [3], while in industrialized countries similar loss percentages (30\%) occur at the retail or consumer levels [1]. The reasons for this massive global food loss are diverse, but microbial spoilage, which affects organoleptic product quality (aspect, texture, taste, and aroma), plays a major role. Among spoilage 
microorganisms, fungi are a major issue at any stage of the food chain because of their ability to grow in different and even harsh environments [4]. Beyond their negative impact on food quality, some fungal genera such as Aspergillus, Penicillium, Alternaria, and Fusarium have the ability to produce secondary metabolites that can have a toxic effect on humans and animals and are therefore named mycotoxins. Moreover, mycotoxins are able to withstand various food processing steps and can thus lead to food safety concerns [5]. Fungi, mainly under an airborne spore form (either sexual or asexual), can settle and grow at different stages of a product life, i.e., at the field, post-harvest, during processing, storage and handling steps by the producer, wholesaler, retailer, and consumer. The impact of contamination at each level can obviously lead to economic losses at both the producer and consumer levels [1]. Moreover, producer losses can be reinforced by a negative brand image induced by consumer dissatisfaction.

In this context, it is crucial to reduce food losses by controlling fungal contamination at all stages of food process chains. Three main stages can be defined to group fungal contamination factors: (i) the field, where water, soil, and air are natural fungal niches; (ii) raw materials—such as post-harvest crops, meats, and milk-where fungal occurrence is related to food management during harvest or collecting, transportation, storage, and packaging [4] and (iii) during food processing while manufacturing dairy, bakery, dry-ripened, and drink products. For field crops, fungal contamination is usually controlled by using synthetic fungicides associated with some crop management practices such as crop rotation, use of resistant cultivars, and tillage [6]. Concerning raw materials, fungicides are widely applied to protect post-harvest fruits and vegetables, but other treatments such as disinfection with ozone, chlorine, acidified hydrogen peroxide, $\mathrm{pH}$ change with sodium bicarbonate, surface sterilization using irradiation or thermal treatments, as well as waxing with active coatings containing fungicidal agents and preservatives are also used. Moreover, different packaging techniques are also used to protect crops from mechanical damage, which is the most common entry point of microbial infections [7,8]. During food processing and raw material storage, good hygiene practices, a hazard analysis critical control point (HACCP) system, efficient equipment and air decontamination, and control of air pressurization to prevent air flow from the dirtiest to cleanest areas-cleanroom technologies (aseptic or ultraclean technologies)—can also be applied to prevent contaminations. Moreover, hurdle technologies are also applied according to the given process and raw materials. They can include thermal treatments such as cooling or heating by pasteurization, sterilization, and heating at ultra-high temperatures (UHT); water removal by drying, freeze-drying, smoking; water activity reduction by addition of high sugar and salt concentrations; $\mathrm{pH}$ modification by immersion in vinegar or other acids; and the use of non-thermal preservation such as modified atmosphere packaging to reduce $\mathrm{O}_{2}$ and increase $\mathrm{CO}_{2}$ levels; and finally high-pressure treatment and high electric field pulse to control or inactivate microorganisms and spores especially in foods and beverages such as yogurts, soups, sauces, liquid eggs, fruit and vegetable juices, raw milk and whey, soft drinks, and alcoholic beverages $[9,10]$. Moreover, chemical preservatives-including benzoate, propionate, sorbate, nitrate, nitrite, and sulfites_are used to avoid fungal spoilage [11]. The only microbial derived antifungal compound used as food preservative is natamycin (E235). It is produced by Streptomyces nataliensis and belongs to the group of polyene macrolide antimycotics. The use of natamycin on cheese as a surface treatment is approved by most countries and its addition into other foods depends on legislation [12].

The potential adverse environmental and health effects of certain fungicides and preservatives have led to more natural methods. Concerning crops, undesirable concentrations of residual pesticides on harvested products, regulations, acquired resistances by fungi, and environmental impact have led to biocontrol agent development (reviewed for fruits and vegetables by [13] and for cereals by [14] currently a fast-growing sector, is also in need of natural solutions to reduce crop losses. Several products composed of bacteria, fungi, and yeasts are currently commercialized worldwide [15-18]. Concerning raw materials and processed foods, preservatives are widely used. However, while their use is regulated in many countries, ingestion of some of them raises questions 
about their potential adverse health effects, especially when considering their chronic consumption over years [11]. Moreover, fungal spoiler resistances have been previously reported in some cases $[19,20]$. In this context, there is a strong societal demand, supported by public authorities, for less processed and preservative-free foods, such as 'clean label' products, and the use of natural alternatives. Such alternatives mainly correspond to the use of essential oils [21-23] and antagonistic microorganisms as preservation tools [24-29]. The use of natural or added microorganisms, fermentates (i.e., an ingredient produced by the fermentation of a variety of raw materials by a microorganism), or their metabolites to extend food shelf-life and/or increase food safety (through antibiosis of pathogenic or spoilage microorganism) is referred to as 'biopreservation' or 'biocontrol' [30]. This alternative approach is complementary to hurdle technologies. Biopreservation by lactic acid bacteria (LAB) is currently the principal alternative to preservatives in food and is widely studied because of the role and long history of use of LAB in fermented foods, their ability to produce antifungal metabolites, and their GRAS (Generally Regarded as Safe) and Qualified Presumption of Safety (QPS) status in the USA and EU, respectively. The use of antifungal LAB for food biopreservation was previously reviewed in 2013 by Crowley [24]. Other antifungal microorganisms to prevent post-harvest fungal spoilage has been reviewed by Sharma et al. (2009) and Liu et al. (2013) [29,31]. Since then, there have been significant advances in the knowledge surrounding the use of antifungal microorganisms. Therefore, the objective of this review is to report the advances on LAB applications as antifungal agents (over the last three years) as well as other antifungal microorganisms, namely non-LAB bacteria, yeasts, and molds that can be used at the post-harvest stage, for raw materials, or during food processing. Crop biocontrol will not be considered in this review and the reader is referred to the recent review for cereals by Oliveira et al. 2014 [14], Punja \& Utkhede, 2003 [32], Milind et al. 2016 [33], and Nguyen et al. 2017 [34]. It will detail the main fungal spoilers encountered in different raw or processed foods, antifungal microorganisms that can be used for food bioprotection, and current knowledge about their mechanisms of action. Finally, advances and constraints associated with the use of antifungal biopreservatives in food and the future areas of research required in this field will be discussed.

\section{Fungal Spoilers}

\subsection{Quality and Safety Issues}

Food products are susceptible to fungal spoilage due to their natural nutrient rich composition. This susceptibility does however depend on various factors, namely (i) the food matrix nature (living material or not, liquid or solid), its composition (nutrient content, solute type), and biological (e.g., its natural microbiota), physical, and chemical parameters (water activity, $\mathrm{pH}$ ); (ii) management during harvesting of fruits and vegetables (maturity, handling) and raw material storage (temperature, hygrometry, and duration); (iii) technological processes applied during manufacture (e.g., heating, drying, salting, fermentation, preservative addition) including cleaning/disinfection steps; and (iv) storage conditions after manufacturing (type, atmosphere and extent of packaging, temperature, relative humidity). Food matrix intrinsic characteristics and also associated extrinsic factors will control the development of certain fungal genera or species. This usually leads to the selection or dominance of one or more fungal species on a given matrix.

Regarding quality, the impact of fungal contaminations can lead to visual and/or other defects. Regarding visual defects, the most obvious is product aspect with apparent fungal growth due to noticeable presence of thallus or yeast colonies that eventually leads to rotting. The presence of black, white, green, pink, or yellow spots can also be associated with fungal development [4]. This usually leads to elimination of the entire product at the industrial or consumer level. According to the nature of the commodity and the contaminant type, the impact might be less evident to observe, but fungal metabolism can lead to various organoleptic defects including gas production, off-flavors, and texture changes. One of the best known off-flavors associated with fungal contamination corresponds to animal-like aroma development linked to volatile phenols (4-ethyl phenol, guaiacol, 
or catechol) in wine and cider by Brettanomyces spp. [35,36]. In wine, molds have also been shown to be responsible for the production of earthy/moldy (e.g., geosmin, methylisoborneol) off-flavors $[37,38]$. Gas production have also been observed. For example, the presence of bubbles was observed in packaged meat inoculated with Kazachstania psychrophila [39]. Kazachstania servazzii has been associated with severe package swelling of prepared fresh pizzas [40] while yogurt has been shown to be contaminated by fermentative yeasts (e.g., Meyerozyma guilliermondii) that led to top lid bulging [41]. Moreover, due to enzymatic lipolytic, proteolytic, and amylolytic activities, product texture can also be affected. For example, Yarrowia lipolytica can produce off-flavors, stimulate the formation of biogenic amines, and negatively affect cheese texture [42].

Regarding safety concerns, fungal presence and growth may also lead to health hazards associated with the presence of mycotoxins that can be produced by species belonging to the Aspergillus, Penicillium, Fusarium, and Alternaria genera. This aspect is of importance as about $25 \%$ of raw materials produced by agriculture worldwide are estimated to be contaminated by fungi and mycotoxins $[43,44]$. Some mycotoxins can induce toxic, carcinogenic, and mutagenic reactions in humans at low concentrations [5]; aflatoxins, ochratoxin A, patulin, fumonisins, zearalenone, T-2 and HT-2 toxins, and deoxynivalenol are considered to be the most significant mycotoxins. The main problem is the presence of mycotoxins in processed foods as the presence of the mycotoxigenic spoilers can no longer be detected, but these metabolites are usually resistant to technological treatments. This has led to regulations on mycotoxin limits in various countries [45]. Among important mycotoxins in food spoilage, aflatoxins and ochratoxin A are produced by several members of Aspergillus while OTA can also be produced by some Penicillium species. Aspergillus flavus, the main aflatoxin producer, is a common spoiler of various commodities (fruits and vegetables, spices, cereals, bread, and nuts such as peanuts, almonds and pistachios). Fumonisins are produced by Fusarium and Aspergillus species. For example, Aspergillus awamori strains infecting onions and Aspergillus niger found in coffee beans and grapes produced fumonisins and ochratoxins. Moreover, a further potential risk is associated with products derived from raw materials containing these mycotoxins [5]. Patulin, which is non-carcinogenic but immunotoxic and neurotoxic in animals, can be produced by several Alternaria, Paelomyces, Aspergillus, and Penicillium species. For example, patulin can be produced by Penicillium expansum, a common apple spoiler, and then be transferred to juices and other derived products (e.g., baby food, apple sauce) [5]. Fungi from the Alternaria genus are common on post-harvest fruit decay. They can produce different mycotoxins such as alternariol, alternariol monomethyl ether, altenuene, altertoxins I, II, III (ATX-I, -II, -III), and tenuazonic acid that have a mutagenic effect [46]. So far, the mycotoxin risk is considered for a single mycotoxin, however, mycotoxin contamination rather corresponds to multi-occurrence due to the simultaneous presence of several mycotoxigenic species and/or of species able to produce several mycotoxins [47].

\subsection{Isolation and Identification of Fungal Spoilers}

Before developing biopreservation or biocontrol tools (i.e., selecting antifungal microbial agents) to control fungal spoilers, it is fundamental to determine the main fungal contaminants and their occurrence in a given food. This mainly relies on their isolation and accurate identification at the species level, which will consequently also provide information on their mycotoxigenic potential. In the context of fungal spoilage, contamination of a given food product is usually linked to a limited number of species and, therefore, culture-dependent methods are the most appropriate. The first step corresponds to isolation and/or enumeration of these microorganisms which can be performed not only from the contaminated food but also from other potential contamination sources like air and surfaces. The use of specific media is a classical step to isolate and determine total counts as well as morphotype observation. After isolation of the observed contaminant, identification is performed using dedicated methods. Although molds can be presumptively identified from their morphology (e.g., by Pitt test), molecular methods have become the gold standard for fungal identification. PCR amplification and sequencing of the D1/D2 domain of the large subunit of ribosomal RNA (26S) and of the internal 
transcribed spacer (ITS) are routinely performed for yeast and molds, respectively [48-51]. However, concerning molds, ITS is not always informative enough for some species, and other gene targets can be sequenced to complete identification at the species level. For example, the $\beta$-tubulin gene is commonly used for Penicillium and Aspergillus spp., actin gene for Cladosporium spp., and the elongation factor gene for Fusarium spp. It is more and more common to use a combination of taxonomically relevant genes to facilitate identification and potentially describe new spoiler agents.

It is worth noting that the constitution of fungal spoiler culture collections would be of great interest to study antifungal cultures. These collections are especially of interest if they contain various strains of a given species. Indeed, most studies only focus on one strain of a given fungal spoiler, raising questions about the representativeness of the selected target strain.

\subsection{Fungal Spoilers and Food Chains}

The diversity of fungi and yeast as food spoilers has been explored in different studies and food products. Notably, fungal species are not always detrimental for food production and some species are even crucial, especially in fermented foods to obtain the typical organoleptic traits of the final product, e.g., Saccharomyces cerevisiae in fermented beverages, Penicillium camemberti in mold-ripened cheeses, or Aspergillus oryzae in soy sauce. Moreover, it should be kept in mind that a fungal species that is expected and often deliberately added in some fermented foods can be a spoiler in another product. For example, Penicillium roqueforti-a species essential for blue-veined cheese production—can spoil grated cheese or fresh cheese.

When considering food spoilage by fungi, two main food categories have been proposed by Pitt \& Hocking, namely (i) fresh or perishable foods (subcategorized in living and non-living cells) and (ii) stored or processed foods [4]. For a complete view of fungal spoilage of these products, one should refer to these authors' books, which are considered as the reference in the domain. Post-harvest fungal spoilage of crops is most often referred to as 'post-harvest disease', and spoilers are referred to as 'pathogens', therefore these terms will also be used hereafter.

\subsubsection{Fresh or Perishable Foods}

Concerning foods and raw materials of plant origin, fruits and vegetables are mainly susceptible to fungal contamination during the ripening stage because of changes in $\mathrm{pH}$, skin, carbohydrates, and defenses that induce favorable conditions for fungal spoilers. Fungi induce visible symptoms on post-harvest crops including discoloration and tissue lesion formation. Their presence leads to spoilage of a variety of fruits including citrus, pome, berry, stone, tropical, and solanaceous fruits. Fungal pathogens of fruits and vegetables have been largely documented [5,8,52]. Tubers and other vegetables-including bulbs, crucifers, cucurbits, and legumes-are less affected than fruits by fungal pathogens, partly because of their $\mathrm{pH}$ making them a more suitable environment for bacterial pathogens [52]. Fungal species belonging to the Penicillium, Botrytis, Monilinia, Rhizopus, Alternaria, Aspergillus, Fusarium, Geotrichum, Gloeosporium, and Mucor genera are responsible for many of the most important postharvest diseases [29].

For foods and raw materials of animal origin, fresh milk and fish are less susceptible to fungal spoilage but highly affected by bacterial spoilage. Concerning meat, fungal spoilage can occur, especially during refrigeration, even if bacterial spoilage is predominant [53]. Despite a need for more studies, Cladosporium, Penicillium, and Aureobasidium species have been reported to provoke black spots, while Thamnidium spp. can cause 'whiskers' on carcasses. Yeast belonging to Cryptococcus, Candida, and Yarrowia genera have been reported on aerobic packaged food and can provoke off-flavors and aspect defects, such as slime or spots [54].

\subsubsection{Stored and Processed Foods}

Stored and processed foods include a wide variety of food products, in which water activity $\left(\mathrm{a}_{\mathrm{W}}\right)$ determines viability and functionality of microrganisms. Most of them can not multiply below 
$0.900 \mathrm{a}_{\mathrm{w}}$, while xerophilic fungi can survive at $0.755-0.605 \mathrm{a}_{\mathrm{w}}$ [55]. Final moisture content and water activity are linked to the technological processes used (e.g., fermentation, drying, salting, evaporation, and freeze-drying) [10]. For intermediate and high water activity products, the main food categories concerned by fungal spoilage are dairy products (yogurt, cream, butter, cheese ... ). These products, usually kept refrigerated, can be affected by both yeasts, especially Candida spp., Yarrowia lipolytica and M. guilliermondii, and molds, mainly Penicillium, Mucor and Cladosporium spp. [49]. Processed meats (chilled meats, bacon), which exhibit an intermediate moisture content, are commonly spoiled by Penicillium, Aerobasidium, Cladosporium, and Eurotium spp., but also Debaryomyces hansenii, Y. lipolytica and Candida spp. For low water activity food products such as cereals, nuts, spices, dried milk, dried meats (dried beef, biltong) and, fermented meats (dry cured ham, salami, and fermented sausage) [4]. Eurotium, Aspergillus, and Penicillium spp. are the main spoilers. Spoiling yeasts such as D. hansenii and Y. lipolytica have also been found in dried meats [56] and Candida spp. in fermented meat [4]. Some of the most xerophilic species are Wallemia sebi, Eurotium repens, Eurotium halophilicum, Xeromyces bisporus, and the yeast Zygosaccharomyces rouxii found in concentrated foods (e.g., fruit concentrates, jams and confectionery), cereals and spices, [55,57,58]. Recently, Aspergillus penicillioides found in dried fish [59] was shown to be the most xerophilic fungal species registered to date as it was able to germinate at $0.585 \mathrm{a}_{\mathrm{w}}[60]$.

Heat treatments, especially when applied to acidic foods, usually allow for fungal destruction. However, ascospores of some species (Byssochlamys nivea) are resistant and can cause spoilage in fruit puree or fruit juices [61]. Noteworthy, contamination of heat-treated products can occur as post-contamination by non-heat-resistant species. This is the case of bakery products, with breads being particularly susceptible to fungal spoilage. The most frequently found genera are Penicillium [62] Aspergillus, Wallemia, and Eurotium [63]. In salted foods, Wallemia sebi, Aspergillus spp. and Eurotium spp. are the main spoilers associated with salted fish [64].

\section{Antifungal Microorganisms in Food}

As previously stated, to answer the strong societal demand for less processed and preservative-free foods, biopreservation has received growing interest for improving food quality and safety $[14,65]$. In past years, many strains from various microbial species that harbor antifungal properties have been identified. They have been isolated from various sources, such as fruits, vegetables, cereals, milk, meat, and other food-related products. The isolation of new bioprotective cultures has recently been extended to other environments, such as deep-sea [66] and Antarctic soil samples [67] in order to discover microorganisms potentially producing new antifungal metabolites.

Screening steps are required to find efficient antifungal microorganisms as antifungal activity levels and the spectrum of the inhibited fungal targets greatly vary depending on the considered species, and from strain to strain within a species. For example, up to $75 \%$ of variation was observed between five strains of Lactobacillus casei tested for their potential to inhibit the growth of four spoilage molds [68]. In another study, only a few L. plantarum isolates among 88 screened showed a wide spectrum of fungi inhibition [69]. In another example, among 55 yeast isolates (Aureobasidium pullulans, Cryptococcus magnus, Hanseniaspora uvarum, Candida zeylanoides, Candida sake, Rhodotorula mucilaginosa, and Pseudozyma aphidis) from the surface of grape varieties, $58 \%$ were able to inhibit $A$. tubingensis growth in vitro and 27 from 37 strains of $A$. pollulans were antifungal indicating a strain-dependent trait [70].

\subsection{Screening and Validation Methods}

\subsubsection{In Vitro Screening}

Candidate bacteria, yeasts, and molds are generally first isolated from various biotopes and purified in order to be tested for their antifungal properties. These candidates are then tested against one or several fungal targets. As stated in the previous chapter, the choice of fungal target(s) is crucial, since they should be representative of the dominant species associated with a given food product and 
since the antifungal effects observed depend on the fungal species. For example, the inhibition of three berry contaminants-namely Botrytis cinerea, Penicillium digitatum and Penicillium italicum-by four antifungal yeasts varied according to the target [71]. Also, growth inhibition of seven targets from the Penicillium, Aspergillus, and Cladosporium genera varied for the 88 Lactobacillus plantarum strains tested [69].

Several approaches, ranging from classical methods in agar plates to high throughput assays in multi well-plates, have been developed to screen microorganisms for their ability to inhibit spoilage fungi. In the spot-on-lawn assay, cultures or cell-free supernatants of the tested strains are spotted over an agar plate, over which a second layer containing the fungal target is poured. In the agar diffusion method, the tested agent is spotted over an agar layer inoculated with the fungal target. In both these assays, formation of an inhibition halo after incubation around the spots reveals the antifungal activity. A dual culture technique has also been used to test the antagonistic effect of a potential antifungal mold against a target mold, in which agar plugs containing the target and the antifungal molds, respectively, were inoculated at the opposite sides over an agar plate [70,72]. Another screening approach corresponds to the investigation of the enzymatic activities required to degrade the cell wall of the fungal target as shown by Tokpah et al. (2016) that screened bacteria for control of a rice pathogen, Magnaporthe grisea. In this context, activity of cell wall degrading enzymes-such as cellulases, proteases, chitinases, and glucanases-was assessed by plating bacteria on agar media containing substrates of these enzymes where active bacteria induced clearing zones [73].

For high-throughput screening purposes, agar plate methods have been recently adapted in 24-well plates. The fungal target can be included in an agar medium and poured in each well over a first agar layer spotted with the potential antifungal strains [74]. Alternatively, spores of the target mold are inoculated on the surface of the first agar layer containing the potential antifungal strains [63].

Most in vitro screening experiments of LAB antifungal activities have been performed using synthetic media, such as the de Man, Rogosa, and Sharpe (MRS) agar medium. The composition of MRS can strongly impact the expression of antifungal activity by LAB because it contains acetate, which may reinforce $\mathrm{LAB}$ antifungal activity and artificially inflate the number of active isolates, as mentioned in several studies $[63,75,76]$. Similarly, the presence of several antifungal compounds-such as cyclic and linear peptides and diketopiperazines-has been reported in the classical lactate-tryptone-yeast extract medium used to grow propionibacteria [77]. Other important points are the nature and concentration of sugars and the buffering capacity of the medium, which are highly expected to influence the amount of organic acids and the final $\mathrm{pH}$ of the medium, thus modifying antifungal activity. Some alternatives to the use of synthetic laboratory media have been recently reported. Particular attention has been paid to design media more closely related to foods and usable in 24-well plates to screen bacterial antifungal activity. This approach was recently used with a wheat flour hydrolysate agar medium and with yogurt [63,78]. Similarly, a method using a model cheese distributed in 24-well plates was also recently developed allowing for high-throughput antifungal activity screening of either LAB fermentates or LAB starters [79].

Whatever the considered screening experiments, it is important to take into account the conditions prevailing in situ to develop relevant screening approaches. Actually, several studies have shown that strains exhibiting in vitro antifungal activity were far less or even no longer active when tested in the food products [63,80-82]. When grown on a wheat flour medium, $20 \%$ of the 270 tested LAB strains representing six genera screened were found to inhibit the five fungal targets tested while none were detected when grown in MRS agar without acetate [63].

For all these reasons, results of in vitro screening are highly heterogeneous in terms of proportion of strains and nature of species harboring antifungal activity. To maximize the chances of finding efficient strains in situ, approaches based on the use of food-related media and screening conditions should be further developed, in particular at a high throughput scale for large collections of strains. 


\subsubsection{Validation by Challenge-Test in the Food Products}

In all cases, in situ evaluation in the actual food products of the antifungal activity of the microorganisms or their metabolites selected from in vitro screening is essential, since, as stated above (see also chapter V), many studies report discrepancies between results observed in vitro and in situ [83].

The developed approaches depend on the type of foods to be protected (raw material or processed food, fermented or not), and on the antifungal microorganism used, to maximize efficiency without adverse effects on the sensory characteristics of the food or on the environment. Biopreservation/biocontrol can be achieved either (i) by adding an active ingredient constituted of a fraction containing purified metabolites, a cell-free supernatant (CFS), a fermentate; or (ii) by using cells expected to grow on or in the food product.

Concerning postharvest fruits, bacterial cells, yeast cells, and cell-free supernatants of molds were evaluated by spraying them onto the surface of an intact fruit or on a wound, before inoculation of the fungal target $[31,84-86]$. Concerning fermented foods, antifungal bacteria can be added as adjunct cultures during the process, along with the usual starters. This has been done in different foods including dairy products [87-89] and bakery products for which adjunct strains were added in the sourdough [90-93]. Alternatively, antifungal ingredients can be incorporated during the process or sprayed over the food, as done for bakery products [63]. In this study, only 12 out of 69 LAB and PAB strains selected from in vitro screening on whey flour agar showed an effect in situ after having sprayed the fermented medium on the surface of the bakery products, thus confirming the necessity to go beyond in vitro tests.

In most cases, fungal targets are inoculated and the ability of tested bioprotective agents to restrain fungal growth is evaluated. Their impact on mycotoxin production can also be investigated, as done for example for deoxynivalenol produced by Fusarium culmorum during malting [94] and aflatoxin produced in vitro by Aspergillus flavus and Aspergillus parasiticus [95]. Environmental challenge tests are also applied in which the activity is tested to prevent environmental fungal contaminants, after exposing the products to the airborne molds of the environment, for example in a bakery [83] or dairy environment [89]. The interest of appropriate in situ tests against a broad range of contaminants has recently been illustrated by studies using antifungal bacteria in bread bioprotection. Amongst the three Lactobacillus strains tested in vitro and in situ, the best performing strain in in situ environmental challenge tests was the least active in vitro [83]. In another study, the antifungal strain L. amylovorus DSM19280 was tested in comparison to a non-antifungal L. amylovorus strain to protect wheat and quinoa sourdough breads and clearly showed that the production of antifungal compounds varied according to the flour type [80].

\subsection{Antifungal Microorganisms}

A large number of microorganisms have been tested for their antifungal activity against food spoilers. This part mainly focuses on the microorganisms successfully tested as bioprotective tools in food products.

Lactic acid bacteria (LAB) are by far the main microorganisms tested for application in dairy and bakery productions, the two main food sectors studied for biopreservation against fungi. These microroganisms have also been tested in brewing (malting process), and in the manufacture of fermented vegetables or to protect grains, seeds, or fruits. For fruit bioprotection, the main candidates tested are bacteria, especially from the Bacillus group, and various yeast species. For fermented meats, yeast (D. hansenii) and Penicillium species have been tested against fungal spoilers. Many of these antifungal species have the characteristics of 'microbial weeds'-i.e., species able to dominate communities that develop in open microbial habitats-a concept recently introduced for microorganisms by Cray et al. 2013 [96]. Table 1 lists the published literature on this subject over the past three years. 
Table 1. Active bioprotective cultures against fungal contaminants of postharvest fruits and processed and raw foods.

\begin{tabular}{|c|c|c|c|c|c|c|c|}
\hline Food Field & Group & $\begin{array}{l}\text { Antifungal Microorganisms } \\
\text { (Active In Situ/Tested Strains) }\end{array}$ & In Situ Test & Source of Microorganism & Application Method & Activity Spectrum (Inhibited/Tested) & Reference \\
\hline bakery & LAB & Lactobacillus brevis ITM18 & yeast-leavened bread & sourdough & CFS as ingredient & Aspergillus niger & [97] \\
\hline bakery & LAB & Lactobacillus plantarum $\mathrm{HD} 1$ & Korean draft rice wine & kimchi & CFS & Aspergillus fumigatus and Pichia kudriavzevii & [98] \\
\hline bakery & LAB & Lactobacillus amylovorus DSM19280 (1/1) & sourdough quinoa bread & cereal isolate & cells in sourdough & environmental molds & [80] \\
\hline bakery & LAB & Lactobacillus plantarum CRL778 & wheat bread & homemade wheat dough & $\begin{array}{l}\text { SL778: fermentate as } \\
\text { ingredient }\end{array}$ & environmental molds & [99] \\
\hline bakery & LAB & $\begin{array}{l}\text { Lactobacillus plantarum UFG } 121 \text { (only } 1 \text { in situ from best } \\
2 / 88 \text { in vitro) }\end{array}$ & oat-based product & food & cells in sourdough & $\begin{array}{l}\text { Fusarium culmorum (only } 1 \text { tested in situ), } \\
\text { Penicillium chrysogenum, Penicillium } \\
\text { expansum, Penicillium roqueforti, and } \\
\text { Aspergillus flavus (5/7 in vitro) }\end{array}$ & [69] \\
\hline bakery & LAB & Lactobacillus amylovorus DSM19280 (1/3) & sourdough wheat bread & cereal isolate & cells as starter & Fusarium culmorum & [83] \\
\hline bakery & LAB & $\begin{array}{l}\text { Lactobacillus reuteri R29, Lactobacillus brevis R2, } \\
\text { Lactobacillus amylovorus DSM19280 }\end{array}$ & $\begin{array}{l}\text { sourdough of quinoa and } \\
\text { rice bread }\end{array}$ & human, pork, and cereal & cells in sourdough & environmental molds & [90] \\
\hline bakery & LAB & $\begin{array}{l}\text { Lactobacillus bulgaricus CECT 4005, Lactobacillus } \\
\text { plantarum CECT } 749 \text { (active in situ 2/6), Lactobacillus } \\
\text { johnsonii } 1 \text { CECT 289, Lactobacillus rhamnosus CECT } 288, \\
\text { Lactobacillis ruminis CECT } 1324 \text {, and Bifidobacterium } \\
\text { bifidum CECT 870T (6 active in vitro/16) }\end{array}$ & loaf bread & not detailed & cells in sourdough & $\begin{array}{l}\text { Aspergillus parasiticus (only one tested } \\
\text { in situ) and Penicillium expansum }\end{array}$ & [100] \\
\hline bakery & LAB \& PAB & $\begin{array}{l}\text { Leuconostoc citreum (5 strains), Lactobacillus sakei, } \\
\text { Lactobacillus plantarum, Lactobacillus spicheri O15, } \\
\text { Lactobacillus reuteri } 5529 \text {, Lactobacillus brevis Lu35, } \\
\text { Propionibacterium acidipropioniciand Propionibacterium } \\
\text { freudenreichii LSaci68 (by surface-spraying } 12 \mathrm{LAB} / 69 \text { ) }\end{array}$ & $\begin{array}{l}\text { pound cake and milk } \\
\text { bread roll }\end{array}$ & $\begin{array}{l}\text { milk, milk roll sourdough, } \\
\text { and others not detailed }\end{array}$ & $\begin{array}{l}\text { whole culture as sourdough } \\
\text { ingredient for milk bread } \\
\text { roll and sprayed }\end{array}$ & $\begin{array}{l}\text { Cladosporium sphaerospermum and Wallemia } \\
\text { sebi on pound cake; and Eurotium repens, } \\
\text { Aspergillus siger, and Penicillium } \\
\text { corylophilum on milk bread roll }\end{array}$ & [63] \\
\hline dairy & LAB & $\begin{array}{l}\text { Lactobacillus harbinensis K.V9.3.1Np, Lactobacillus. } \\
\text { rhamnousus K.C8.3.11, and Lactobacillus paracasei } \\
\text { K.C8.3.1Hc1 (3/11) }\end{array}$ & yogurt & cow and goat milk & cells as adjunct culture & $\begin{array}{l}\text { Debaryomyces hansenii, Kluyveromyces lactis, } \\
\text { Kluyveromyces marxianus, Penicillium } \\
\text { brevicompactum, Rhodotorula mucilaginosa, } \\
\text { and Yarrowia lipolytica }(6 / 6)\end{array}$ & [78] \\
\hline dairy & LAB & Lactobacillus casei AST18 (1/1) & yogurt & Chinese dairy products & cells as adjunct culture & Penicillium sp. (1/1) & [88] \\
\hline dairy & LAB & Lactobacillus paracasei DCS302 & yogurt & not detailed & cells as adjunct culture & $\begin{array}{l}\text { Penicillium sp. nov. DCS 1541, Penicillium } \\
\text { solitum (2/8) }\end{array}$ & [101] \\
\hline dairy & LAB & Lactobacillus harbinensis K.V9.3.1Np (1/2) & yogurt & cow milk & cells as adjunct culture & Yarrowia lipolytica (1/1) & [87] \\
\hline dairy & LAB & Lactobacillus reuteri INIA P57 & semi-hard ewe milk cheese & $\begin{array}{l}\text { pig feces (isolated by } \\
\text { Langa 2003) }\end{array}$ & $\begin{array}{l}\text { cells as adjunct culture } \\
\text { supplemented with glycerol }\end{array}$ & Not evaluated & [102] \\
\hline dairy & LAB & Lactobacillus amylovorus DSM 19280 (1/1) & cheddar cheese & cereal environment & cells as adjunct culture & $\begin{array}{l}\text { Penicillium expansum ( } 1 / 1) \text { and } \\
\text { environmental molds }\end{array}$ & [89] \\
\hline dairy & LAB & 12 strains of Lactobacillus plantarum (12/897) & cottage cheese & $\begin{array}{l}\text { fresh herbs, fruits, } \\
\text { and vegetables }\end{array}$ & $\begin{array}{l}\text { cells as added to the } \\
\text { finished product }\end{array}$ & Penicillium commune & [103] \\
\hline dairy & LAB & $\begin{array}{l}\text { L. rhamnosus A238, L. rhamnosus A119 }(2 / 5) \text { The } \\
\text { association of L. rhamnosus A238 with B. animalis subsp. } \\
\text { lactis A026, and L L rhamnosus A119 with B. animalis } \\
\text { subsp. lactis A026 }\end{array}$ & cottage cheese & not detailed & $\begin{array}{l}\text { cells added to the } \\
\text { finished product }\end{array}$ & Penicillium chrysogenum (1/1) & [104] \\
\hline malting & LAB & Lactobacillus brevis R2 $\Delta(1 / 1)$ & $\begin{array}{l}\text { barley malt extract } \\
\text { fermentation }\end{array}$ & porcine isolate & cells as starter & Not evaluated & [105] \\
\hline
\end{tabular}


Table 1. Cont.

\begin{tabular}{|c|c|c|c|c|c|c|c|}
\hline Food Field & Group & $\begin{array}{l}\text { Antifungal Microorganisms } \\
\text { (Active In Situ/Tested Strains) }\end{array}$ & In Situ Test & Source of Microorganism & Application Method & Activity Spectrum (Inhibited/Tested) & Reference \\
\hline malting & LAB & $\begin{array}{l}\text { Lactobacillus brevis R2 } \Delta \text { and Lactobacillus plantarum } \\
\text { FST1.7 (2/2) }\end{array}$ & $\begin{array}{l}\text { barley malt extract (wort) } \\
\text { fermentation }\end{array}$ & porcine and barley isolate & cells as starter & Fusarium culmorum & [106] \\
\hline malting & LAB & Lactobacillus brevis R2 $2(1 / 1)$ & barley in malting process & porcine isolate & cells as starters and CFS & $\begin{array}{l}\text { Fusarium culmorum and } \\
\text { Fusarium graminearum }\end{array}$ & [107] \\
\hline malting & LAB & $\begin{array}{l}\text { Lactobacillus reuteri R29 and Lactobacillus amylovorus } \\
\text { DSM19280 }\end{array}$ & $\begin{array}{l}\text { malting process (steeping } \\
\text { and germination) }\end{array}$ & human and cereal isolates & $\begin{array}{l}\text { CFS (wort as growth media) } \\
\text { as the steeping liquor }\end{array}$ & Fusarium culmorum & [94] \\
\hline $\begin{array}{l}\text { fermented } \\
\text { vegetables }\end{array}$ & LAB & $\begin{array}{l}\text { Pediococcus spp. A19 (tested in situ) Pediococcus spp. } \\
\text { A21, Lactobacillus plantarum B4496, Lactobacillus brevis } \\
\text { 207, and Lactobacillus sanfranciscensis BB12 (5/13) }\end{array}$ & cocoa & fermenting cocoa & cells as starter & $\begin{array}{l}\text { Aspergillus carbonarius, Aspergillus niger, } \\
\text { and Aspergillus ochraceus }\end{array}$ & [108] \\
\hline $\begin{array}{l}\text { fermented } \\
\text { vegetables }\end{array}$ & LAB & Lactobacillus fermentum YML014 & tomato puree & $\begin{array}{l}\text { gari, fermented cassava } \\
\text { (starchy root) }\end{array}$ & cells & $\begin{array}{l}\text { Penicillium expansum (only one tested in } \\
\text { situ), Aspergillus flavus, Aspergillus niger, } \\
\text { Candida albicans, and Zygosaccharomyces } \\
\text { rouxii (low inhibition of yeasts) }\end{array}$ & [109] \\
\hline $\begin{array}{l}\text { fermented } \\
\text { vegetables }\end{array}$ & LAB & $\begin{array}{l}\text { Lactobacillus helveticus KLDS } 1.8701 \text { (1/4 also } L \text {. } \\
\text { helveticus) }\end{array}$ & fermented soybean milk & dairy products & cells as adjunct culture & Penicillium sp. $(1 / 1)$ & [81] \\
\hline grain/seed & LAB & Lactococcus sp. BSN307 & wheat grains & $\begin{array}{l}\text { rotten jackfruit, guava, and } \\
\text { animals fecal samples }\end{array}$ & $\begin{array}{l}\text { submerged in purified } \\
\text { volatile organic compound } \\
\text { 2,4-di-tert-butylphenol }\end{array}$ & $\begin{array}{l}\text { A. niger, F. moniliforme, F. graminearum, } \\
\text { F. chlamydosporum, and F. oxysporum }\end{array}$ & [110] \\
\hline grain/seed & LAB & Lactobacillus plantarum LR/14 & $\begin{array}{l}\text { wheat seeds (Triticum } \\
\text { aestivum var. HD 2824) }\end{array}$ & not detailed & AMP LR14 solution & $\begin{array}{l}\text { Aspergillus niger, Rhizopus stolonifer, Mucor } \\
\text { racemosus, and Penicillium chrysogenum. }\end{array}$ & [111] \\
\hline grain/seed & LAB & Lactobacillus plantarum YML007 (1/1400) & soybean & kimchi & CFS & Aspergillus niger & [112] \\
\hline grain/seed & $\begin{array}{l}\text { LAB \& } \\
\text { Fungi }\end{array}$ & $\begin{array}{l}\text { kefir grains contain a symbiotic consortium of LAB and } \\
\text { yeasts (Lactobacillus plantarum, L. kefir, Lactococcus lactis } \\
\text { subsp. lactis, Saccharomyces and Acetobacter) }\end{array}$ & arepa (corn cakes) & kefir grains & CFS & Aspergillus flavus & [113] \\
\hline fruit & $\begin{array}{l}\text { LAB \& } \\
\text { other } \\
\text { bacteria }\end{array}$ & $\begin{array}{l}\text { Lactobacillus lactis subsp. lactis LABW1, LABW3, } \\
\text { LABW4, Burkholderia cenocepacia VBC7 and } \\
\text { Pseudomonas poae VBK1 }\end{array}$ & jackfruit & rotten jackfruit & cells sprayed over the fruit & Rhizopus stolonifer & [114] \\
\hline fruit & LAB & $\begin{array}{l}\text { Lactobacillus paracasei ŁOCK0921 (1 tested in situ) } \\
(1 /(9 \text { in vitro/60)) }\end{array}$ & wild cherries & plant and human & $\begin{array}{l}\text { CFS cultivated with xylitol } \\
\text { or galactosyl-xylitol directly } \\
\text { on wild cherries }\end{array}$ & $\begin{array}{l}\text { Alternaria brassicicola ( } 1 \text { tested in situ), } \\
\text { Alternaria alternata, Aspergillus niger, } \\
\text { Fusarium lateritium, Geotrichum candidum, } \\
\text { and Mucor hiemalis (6/8) }\end{array}$ & [115] \\
\hline fruit & LAB & $\begin{array}{l}\text { Lactic acid bacteria strains LCM5, LAB } 58 \text {, LAB 13, } \\
\text { and LAB } 43(4 / 6)\end{array}$ & apple & $\begin{array}{l}\text { plants, fermented wheat } \\
\text { bran, pickles, and } \\
\text { sauerkraut }\end{array}$ & $\begin{array}{l}\text { cells sprayed over the fruit } \\
\text { (wounded and } \\
\text { non-wounded) }\end{array}$ & Penicillium expansum & [116] \\
\hline fruit/dairy & LAB & Lactobacillus plantarum TK9 & citrus, apples and yogurt & $\begin{array}{l}\text { Chinese naturally } \\
\text { fermented congee }\end{array}$ & cells & $\begin{array}{l}\text { Penicillium roqueforti, Penicillium citrinum, } \\
\text { Penicillium oxalicum, Aspergillus fumigatus, } \\
\text { Aspergillus flavus, and } \\
\text { Rhizopus nigricans }(6 / 7)\end{array}$ & [117] \\
\hline grain/seed & $\begin{array}{l}\text { Other } \\
\text { bacteria }\end{array}$ & Acetobacter nigricans AZT $54(0 / 1)$ & $\begin{array}{l}\text { maize, sorghum and } \\
\text { wheat grains }\end{array}$ & paddy field soil samples & $\begin{array}{l}\text { cereals submerged } \\
\text { in suspension }\end{array}$ & $\begin{array}{l}\text { Fusarium sporotrichioides, Fusarium } \\
\text { graminearum, Fusarium poae, and Fusarium } \\
\text { equiseti ( } 4 / 10 \text { in vitro) }\end{array}$ & [82] \\
\hline
\end{tabular}


Table 1. Cont.

\begin{tabular}{|c|c|c|c|c|c|c|c|}
\hline Food Field & Group & $\begin{array}{l}\text { Antifungal Microorganisms } \\
\text { (Active In Situ/Tested Strains) }\end{array}$ & In Situ Test & Source of Microorganism & Application Method & Activity Spectrum (Inhibited/Tested) & Reference \\
\hline grain/seed & $\begin{array}{l}\text { Other } \\
\text { bacteria }\end{array}$ & Bacillus cereus & peanut kernels & $\begin{array}{l}\text { entomopathogenic } \\
\text { nematode }\end{array}$ & $\begin{array}{l}\text { cells and purified } \\
\text { cyclo(4-hydroxy-L-Pro-L-Trp) }\end{array}$ & Aspergillus flavus & [118] \\
\hline fruit & $\begin{array}{l}\text { Other } \\
\text { bacteria }\end{array}$ & Bacillus subtilis AFB22 [1/(50/200 in vitro)] & pomegranate & $\begin{array}{l}\text { pomegranate leaves and } \\
\text { fruits }\end{array}$ & $\begin{array}{l}\text { cells and CFS by spraying } \\
\text { on wounded fruits }\end{array}$ & $\begin{array}{l}\text { P. varsoniana (only in situ), A. flavus, } \\
\text { A. clavatus, B. humicola, F. graminearum, } \\
\text { and R. stolonifer (in vitro) }\end{array}$ & [119] \\
\hline fruit & $\begin{array}{l}\text { Other } \\
\text { bacteria }\end{array}$ & Bacillus amyloliquefaciens ZJ01 and ZJ02 & jujube fruit & $\begin{array}{l}\text { phyllosphere of } \\
\text { Chinese jujube }\end{array}$ & $\begin{array}{l}\text { whole culture in created } \\
\text { wounds-prevention } \\
\text { treatment }\end{array}$ & $\begin{array}{l}\text { Phoma destructiva (2 strains), Alternaria } \\
\text { alterna (2 strains), and } \\
\text { Fusicoccum spp. }(5 / 5)\end{array}$ & [120] \\
\hline fruit & $\begin{array}{l}\text { Other } \\
\text { bacteria }\end{array}$ & Bacillus subtilis V26 & tomato fruit & rhizosphere of almond trees & $\begin{array}{l}\text { whole culture, endospores, } \\
\text { and CFS in created wounds }\end{array}$ & Botrytis cinerea & [84] \\
\hline fruit & $\begin{array}{l}\text { Other } \\
\text { bacteria }\end{array}$ & $\begin{array}{l}\text { Paenibacillus polymyxa APEC136 and Bacillus subtilis } \\
\text { APEC170 }\end{array}$ & apple & $\begin{array}{l}\text { soil from several } \\
\text { apple orchards }\end{array}$ & cells over created wounds & $\begin{array}{l}\text { Colletotrichum gloesporioides, Colletotrichum } \\
\text { acutatum, and Botryosphaeria dothidea }\end{array}$ & [121] \\
\hline fruit & $\begin{array}{l}\text { Other } \\
\text { bacteria }\end{array}$ & Bacillus amyloliquefaciens BUZ-14 & $\begin{array}{l}\text { apple, orange, grape, } \\
\text { and cherries }\end{array}$ & $\begin{array}{l}\text { surface of peach fruit from } \\
\text { an orchard }\end{array}$ & cells, endospores and CFS & $\begin{array}{l}\text { Botrytis cinerea, Monilinia fructicola, } \\
\text { Monilinia laxa, Penicillium digitatum, } \\
\text { Penicillium expansum, and } \\
\text { Penicillium italicum }\end{array}$ & [122] \\
\hline fruit & $\begin{array}{l}\text { Other } \\
\text { bacteria }\end{array}$ & Cryptococcus laurentii & peach fruit & surfaces of apple fruits & cells in created wounds & Penicillium expansum & [123] \\
\hline fruit & $\begin{array}{l}\text { Other } \\
\text { bacteria }\end{array}$ & Bacillus amyloliquefaciens CPA-8 & cherries & nectarine surface & $\begin{array}{l}\text { wounded fruits packaged } \\
\text { with in situ produced } \\
\text { volatile organic compounds }\end{array}$ & Monilia fructicola $(1 / 3)$ & [124] \\
\hline fruit & $\begin{array}{l}\text { Other } \\
\text { bacteria }\end{array}$ & Paenibacillus pasadenensis R16 & grape berries & leaf of grapevine plant & $\begin{array}{l}\text { wounded fruit submerged } \\
\text { in cell suspension }\end{array}$ & Botrytis cinerea & [125] \\
\hline fruit & Yeast & Cryptococcus laurentii 2.3803 & strawberries & not detailed & $\begin{array}{l}\text { cells sprayed over fruits } \\
\text { prior to harvest }\end{array}$ & Botrytis cinerea & [126] \\
\hline fruit & Yeast & Hanseniaspora uvarum & grape berries & strawberries surface & $\begin{array}{l}\text { cells in wounds and fruit } \\
\text { submerged in salicylic acid } \\
\text { or sodium bicarbonate }\end{array}$ & Botrytis cinerea & [127] \\
\hline fruit & Yeast & $\begin{array}{l}\text { Wickerhamomyces anomalus BS91, Metschnikowia } \\
\text { pulcherrima MPR3, Aureobasidium pullulans PI1, and } \\
\text { Saccharomyces cerevisiae BCA62 }\end{array}$ & grape berries & $\begin{array}{l}\text { fermented olive brine and } \\
\text { pomegranate }\end{array}$ & cells in created wounds & Botrytis cinerea & [71] \\
\hline fruit & Yeast & $\begin{array}{l}\text { Aureobasidium pullulan (25 strains), Cryptococcus magnus } \\
\text { (2 strains), Candida sake } 2 \mathrm{AM} 3 \text { [(28/33 in situ) } \\
(33 / 55 \text { in vitro)] }\end{array}$ & grape berries & surface of grape berries & $\begin{array}{l}\text { wounded fruits submerged } \\
\text { in cells suspension }\end{array}$ & Aspergillus tubingensis & [70] \\
\hline fruit & Yeast & Candida intermedia and Wickerhamomyces anomalus & avocado & $\begin{array}{l}\text { fruits, leaves, and the soil of } \\
\text { the avocado orchards }\end{array}$ & cells in created wounds & $\begin{array}{l}\text { Colletotrichum gloeosporioides and } \\
\text { Colletotrichum acutatum }\end{array}$ & [128] \\
\hline fruit & Yeast & Hanseniaspora uvarum $\mathrm{Y} 3$ & orange & $\begin{array}{l}\text { surfaces of grapes } \\
\text { in vineyard }\end{array}$ & cells in created wounds & Penicillium digitatum & [85] \\
\hline fruit & Yeast & Pichia membranaefaciens & citrus fruits Citrus sinensis & not detailed & cells in created wounds & Colletotrichum gloerosporioides & [129] \\
\hline fruit & Yeast & $\begin{array}{l}\text { Rhodotorula minuta ACBL-23, Candida azyma ACBL-44, S. } \\
\text { cerevisiae ACBL-52, Rhodotorula mucilaginosa ACBL-68, } \\
\text { and Aureobasidium pullulans ACBL-77 }\end{array}$ & 'Pera' orange fruits & $\begin{array}{l}\text { citrus leaves, flowers, fruits, } \\
\text { and citrus-growing soils }\end{array}$ & cells in created wounds & Geotrichum citri-aurantii & [130] \\
\hline
\end{tabular}


Table 1. Cont.

\begin{tabular}{|c|c|c|c|c|c|c|c|}
\hline Food Field & Group & $\begin{array}{l}\text { Antifungal Microorganisms } \\
\text { (Active In Situ/Tested Strains) }\end{array}$ & In Situ Test & Source of Microorganism & Application Method & Activity Spectrum (Inhibited/Tested) & Reference \\
\hline fruit & Yeast & $\begin{array}{l}\text { Pichia fermentans (2 strains), Wickerhamomyces anomalus, } \\
\text { Kazachstania exigua, and Saccharomyces cerevisiae }\end{array}$ & lemons & $\begin{array}{l}\text { surface of leaves and fruits } \\
\text { of different citrus and } \\
\text { wash-water from } \\
\text { lemon shells }\end{array}$ & $\begin{array}{l}\text { wounded fruits submerged } \\
\text { cells suspension }\end{array}$ & $\begin{array}{l}\text { Penicillium digitatum and } \\
\text { Penicillium italicum }\end{array}$ & [131] \\
\hline fruit & Yeast & $\begin{array}{l}\text { Debaryomyces hansenii KI2a, D. hansenii MIIa, and } \\
\text { Wickerhamomyces anomalus BS91 }\end{array}$ & peach and plum fruits & $\begin{array}{l}\text { blue-veined Rokpol cheese } \\
\text { and fermented olive brine }\end{array}$ & cells in created wounds & Monilinia fructigena and Monilinia fructicola & [132] \\
\hline fruit & Yeast & Candida tropicalis YZ27 & banana & from bitter gourd & cells in created wounds & Colletotrichum musae & [133] \\
\hline fruit & Yeast & Yarrowia lipolytica & grape berries & surface of grapes & cells in created wounds & Talaromyces rugulosus & [134] \\
\hline meat & Yeast & Debaromyces hansenii FHSCC 253H & dry-cured ham slices & dry-cured meat products & $\begin{array}{l}\text { cells over slices } \\
\text { ( } \mathrm{a}_{\mathrm{w}} \text { controlled) }\end{array}$ & Penicillium nordicum & [135] \\
\hline meat & Yeast & Debaromyces hansenii $253 \mathrm{H}$ and $226 \mathrm{G} \mathrm{G}$ & dry-fermented sausage & dry-cured meat products & $\begin{array}{l}\text { cells over slices after } \\
\text { fermentation }\end{array}$ & Penicillium verrucosum & [136] \\
\hline meat & Molds & Penicillium nalgoviense & dry-fermented sausages & $\begin{array}{l}\text { TEXEL PN1 from Danisco } \\
\text { (Niebüll, Germany) }\end{array}$ & $\begin{array}{l}\text { immersion of sausages in } \\
\text { cells suspension }\end{array}$ & Penicillium verrucosum & [137] \\
\hline meat & Molds & Penicillium chrysogenum CECT 20922 & dry-cured ham slices & not detailed & cells & $\begin{array}{l}\text { Cladosporium cladosporioides, C. herbarum, } \\
\text { and C. oxysporum }\end{array}$ & [138] \\
\hline fruit & Molds & Clonostachys rosea & tomato fruit & not detailed & cells sprayed over the fruit & Botrytis cinerea & [139] \\
\hline
\end{tabular}




\subsubsection{Lactic Acid Bacteria}

LAB encompasses a large and heterogeneous group of Gram-positive, low-GC, acid-tolerant bacteria, which produce lactic acid as the major metabolic end product of carbohydrate fermentation. LAB belong to the Lactobacillales order, which include 6 families, 36 genera, and more than 200 species. They are found in various biotopes such as environment, plants, human, and animal microbiota. They are largely used in the manufacture of a variety of fermented foods, where they contribute to improve shelf-life, organoleptic properties, and nutritional value. The main LAB in fermented foods correspond to species belonging to the Lactobacillus, Lactococcus, Leuconostoc, Carnobacterium, Enterococcus, Oenococcus, Pediococcus, Streptococcus, Tetragenococcus, Vagococcus, and Weissella genera [28].

Lactobacillus, Pediococcus, and Leuconostoc species have been the most studied for their antifungal activity $[24,25,140]$ and they have also been the most evaluated in situ in the past years (Table 1 ). Among them, L. plantarum is the most studied species representing about one third of reports on LAB and many different strains have been tested as antifungals in foods since 2013, (Table 1). L. plantarum strains and their metabolites have been tested in a wide range of foods where they inhibited different fungal species such as Aspergillus, Penicillium, Rhizopus, Rhodotorula, and Pichia spp. L. plantarum is a ubiquitous species [141] and is found in a wide range of ecological niches, such as milk [75], water for malt production[142], malted barley [143], or the aerial surfaces of plants or vegetables [33]. L. plantarum is also widely present in fermented foods, including different cheese varieties [144], and thus has the potential to be tested as an antifungal culture in a variety of applications, as already shown for the strain L. plantarum TK9 active against Penicillium in citrus, apple, and yogurt spoilage [117]. In another recent study, L. plantarum UFG 121 retarded the growth of F. culmorum in an oat-based beverage fermented with this strain [69]. There is a growing interest in looking for new antifungal strains in diverse types of fermented foods. With this in mind, L. plantarum strains with antifungal activities have been isolated from a variety of fermented foods including kimchi [98,112], koumiss[144], tempeh [145], and a number of other traditional fermented vegetables. $[95,146]$.

Other species of lactobacilli have also recently been identified as potential antifungal cultures, such as L. rossiae[147], L. amylovorus [148], L. harbinensis [78], L. brevis, and L. spicheri [63]. Many other Lactobacillus species-including L. rhamnosus, L. casei, L. paracasei, L. sanfranciscensis, L. fermentum, L. helveticus, and $L$. sakei-added as adjunct cultures in fermented foods, have been shown to be able to extend the shelf life of various products: yogurt [87-89,142], fermented drinks [98] and bakery products [83,97].

Leuconostoc have also shown antifungal activities. Leuconostoc are used as starters in some fermented dairy products [149] but are also natural contaminants in various food products such as cheese and modified atmosphere packaged meat and seafood, where they can be responsible for spoilage [150]. In bakery products, Leuconostoc spp. exhibited good antifungal potential against fungal spoilers, with the highest proportion of active isolates against the 5 tested fungal targets, and 5 strains of L. citreum among the 10 most active LAB strains selected [63].

Concerning pediococci, several studies showed in vitro activity of Pediococcus pentosaceus strains against Aspergillus flavus and Aspergillus niger [151]. For example, 5 strains including 2 pediococci and 3 lactobacilli were selected from in vitro assessment of 13 strains based on their antifungal properties towards three ochratoxin A-producing Aspergillus species. One Pediococcus spp. strain was selected to be used as starter in cocoa fermentation inoculated with $A$. carbonarius, where it reduced fungal populations and toxin production [108].

Candidate antifungal bacteria to be tested in a given food product are often chosen among strains isolated from a similar biotope. However, successful results have also been obtained with strains tested in applications unrelated to their original biotope. For example, L. amylovorus DSM19280, a species commonly isolated from cereals or whole barley sourdough, inhibited the growth of F. culmorum that grows during the malting process and deoxynivalenol production, a mycotoxin produced by this common plant pathogen [152]. This LAB species also performed well in a bakery environmental challenge test [83]. Interestingly, the same L. amylovorus strain, added as an adjunct culture along with the usual lactic starters in Cheddar cheese manufacture, delayed Penicillium expansum growth 
by four days during ripening [89]. Similarly, a L. reuteri strain R29 isolated from human microbiota, was successfully used during the malting process to inhibit F. culmorum [94], and to extend the shelf life in rice and quinoa breads by incorporating L. reuteri in the sourdough [90]. These examples suggest that candidate strains selected for their antifungal properties from screening procedures could also be chosen without restriction of biotopes.

\subsubsection{Propionibacteria}

Dairy propionibacteria (PAB) are also considered as antifungal candidates. The Propionibacterium genus, belonging to the Actinobacteria class, is a Gram-positive, high-GC content bacteria divided into 'cutaneous' and 'classical' (also referred to as 'dairy') propionibacteria based on their main isolation biotopes [153]. PAB and related species taxonomy has recently been reconsidered, with in particular 'dairy' PAB separated in the Propionibacterium genus consisting of $P$. freudenreichii, the main species used in cheese-making, and three other species, and a novel genus-Acidipropionibacterium-which encompasses the former species P. acidipropionici, P. thoenii, P. jensenii, and three other species [141].

In a large in vitro screening study on 197 dairy PAB strains, 13 strains-including 9 P. jensenii, 2 P. acidipropionici, and 2 P. thoenii-showed high antifungal activity against various yeast and mold species. In another study, in vitro screening revealed that almost all PAB strains were active in vitro against five mold species. Only two strains of $P$. acidipropionici and P. freudenreichii slightly delayed A. niger and $P$. corylophilum growth in milk bread rolls sprayed with antifungal cultures, whereas no inhibitory effect was observed in pound cake, probably because of the near neutral $\mathrm{pH}$ of this bakery product [63].

\subsubsection{Bacillus and Other Bacteria}

Bacilli are Gram-positive, aerobic endospore-forming, and rod-shaped bacteria. They are found in diverse environments and are known to produce a variety of secondary metabolites including antimicrobial compounds. Their antimicrobial activities have been applied for the development of medical treatments but also more recently, as biocontrol agents of pre-harvest crop diseases and postharvest fruit and vegetable spoilage. In particular, Bacillus subtilis strains produce a wide variety of antimicrobial compounds, which include peptides and non-peptides [154].

Some recent bioprotection assays performed in situ using Bacillus species cells, endospores, CFS, or purified peptides on postharvest fruits illustrate the potential activities that they can exhibit (Table 1). Bacillus sp. strains present a large activity spectrum against pathogenic fruit molds from the Aspergillus, Alternaria, Beltraniella, Botryosphaeria, Botrytis, Monilinia, Colletotrichum, Fusarium, Fusicoccum, Penicillium, Phomopsis, Phoma, Rhizoctonia, and Rhizopus genera. Bioprotective activities were demonstrated in situ in a variety of fruits including tomatoes, apples, grapes, and pomegranates (Table 1). For example, B. subtilis AFB22 cells and CFS, selected after screening 200 antifungal Bacillus spp. isolates, prevented pomegranates from rotting when challenged with spores of Phomopsis varsoniana [119]. In another study, whole cultures of two B.amyloliquefaciens strains sprayed on Chinese jujube fruit inoculated with spores of three key pathogens of this fruit, Phoma destructiva, Alternaria alternata and Fusicoccum spp., reduced the disease incidence and induced different kinds of hyphal alterations on the fungal targets [120]. In vitro test using CFS from B. amyloliquefaciens also inhibited the growth of B. cinerea, a postharvest phytopathogen [155] and Botryosphaeria dothidea involved in peach gummosis [156].

Some Bacillus cereus sensu lato species have been recently proposed as bioprotective cultures and correspond to species commonly found in soil and foods but they may also be potential spoilers in dairy products and cereals and in some cases, harmful to humans. A B. cereus sensu lato strain isolated from an entomopathogenic nematode and its metabolites were active against an Aspergillus sp. in peanuts [118].

\subsubsection{Yeasts}

If bacteria are well known for producing a large diversity of antimicrobial compounds, in recent decades, there has been a significant increase in interest regarding yeast antimicrobial properties. 
Yeasts are single cell microorganisms classified as members of the fungi kingdom. They are ubiquitous in the environment, are able to grow in a large variety of biotopes such as cereals, vegetables, fruits, meat, milk, as well as processed food products. Yeasts have been involved in food preservation for millennia, through the fermentation process of wine, beer, cereal-doughs, and certain cheese varieties, [157] where they contribute to organoleptic properties of fermented foods. Their antimicrobial activity is attributed to their ability to compete for nutrients, to acidify the medium, to resist stressful conditions (ethanol), but also to produce antimicrobial molecules named 'mycocins', also referred to as killer proteins affecting fungal growth [158]. Moreover, their capacity to colonize fruit, seeds, berries, leaves, and to compete for space and nutrients with other microorganisms, make them good candidates as biocontrol agents to limit postharvest decay for example [159]. Yeast antimicrobial activities and the numerous applications of mycocin-producing yeasts for preventing fungal spoilage in various foods and beverages such as wine, olives, beer, sake, miso, soy sauce, and salted vegetables have been reviewed by different authors (e.g. [160]). Nevertheless, these authors underlined that yeast strains must be used with caution since they could have a negative impact on the quality of end-products.

The yeasts Meyerozyma guilliermondii, Candida fructus, Issatchenkia orientalis, and Candida quercitrusa are frequently found associated with fruits or plant surfaces and showed antagonistic activities against fungal pathogens [161]. In a study on 11 yeast strains isolated from avocados, one Wickerhamomyces anomalus strain was able to inhibit Colletotrichum gloeosporioides and C. acutatum responsible for avocado anthracnose [128]. In the case of citrus fruit anthracnose, Pichia membranifaciens-alone or in combination with chitosan-inhibited C. gloeosporioides mycelium growth and spore germination [129]. Hanseniaspora uvarum isolated from grape surfaces has been reported as a natural preservative acting against the disintegration of gray post-harvest grapes caused by B. cinerea [127]. The same species was active against the green mold P. digitatum that spoils citrus fruits [85].

Antifungal yeast applications have also been suggested in the past three years for the production of dry-cured ham [136] and sausages [135] to control toxigenic penicillia populations that lead to spoilage inducing visual (black spots) and flavor defects, and above all that can produce ochratoxin A (OTA). In these studies, two $D$. hansenii strains revealed antifungal activity against Penicillium verrucosum and P. nordicum and were able to decrease OTA production. Paradoxically, D. hansenii can be involved in food spoilage as shown for some dairy products such as fresh cheese or cream but, at the same time, is food grade and used as a starter for specific dairy products. It is naturally found in meat products and on fruit surfaces and is used as a ripening culture to manufacture some surface ripened cheeses.

Foods are not the sole source of potential bioprotective yeasts, and yeasts with an antifungal potential have also been isolated from marine environments. This ecosystem confers unique properties to yeast matching with their potential use as bioprotective agents for postharvest fruits and vegetables. Antimicrobial activity screening of deep-sea fungi from the South China sea showed that $56 \%$ of fungal isolates exhibited antimicrobial activity against at least one pathogenic bacterium or fungus. Out of these antimicrobial fungi, the genera Arthrinium, Aspergillus, and Penicillium exhibited antibacterial and antifungal activities, while genera Acremonium, Cladosporium, Geomyces, and Phaeosphaeriopsis displayed only antifungal activity [162]. The marine yeast, Rhodosporidium paludigenum, isolated from the East China Sea, effectively inhibited P. expansum on pear fruit, and Alternaria alternata on jujube fruits [163]. A number of bioactive metabolites produced by deep-sea fungi have been recently reviewed, thus more than 180 bioactive secondary metabolites derived from deep-sea fungi have been documented in the literature, including compounds with antifungal activity [66].

\subsubsection{Filamentous Fungi}

As previously stated, not all filamentous fungi are responsible for food spoilage and postharvest crop disease. Some of them contribute to the flavor and typical visual characteristics of some fermented foods—such as surface-ripened cheese [164], fermented sausage [50], and ham [138]—and can also be used as antifungal agents, in particular to protect the surface of dry-ripened products where the low $\mathrm{a}_{\mathrm{w}}$ prevent the use of antifungal LAB. Some filamentous fungi such as the Penicillium species 
P. nalgiovense (non-toxigenic) and P. chrysogenum are both commercial cultures commonly used in the meat industry [165]. Both species have recently been tested against Aspergillus and Penicillium mycotoxin producers. P. chrysogenum strains isolated from dry-cured ham inhibited two common spoilers A. flavus and P. restrictum in vitro [166,167] by the production of an antifungal protein, whereas P. nalgoviense, from the commercial culture TEXEL PN1, limits the growth and OTA production of P. verrucosum [137]. In another study of the bioprotective role of $P$. chrysogenum, it was suggested to combine the use of $P$. chrysogenum CECT 20922 as bioprotective culture and the reduction of $\mathrm{a}_{\mathrm{w}}$ throughout the ripening process to avoid black spot formation in dry-cured ham [138].

As will be detailed below, fungi produce antimicrobial peptides (AMP) that are a valuable strategy to avoid fungal spoilage and mycotoxin production.

\section{Action Mechanisms of Antifungal Microorganisms}

Fungal spoilage control using antifungal microorganisms is a complex task which success depends on network of the interactions between three main actors: the food itself (comprising its natural microbiota), the fungal spoiler(s), and the antifungal microorganism(s) (Figure 1). Depending on food type and antifungal microorganism, different action mechanisms—combined or not-can be responsible for spoilage fungi inhibition.

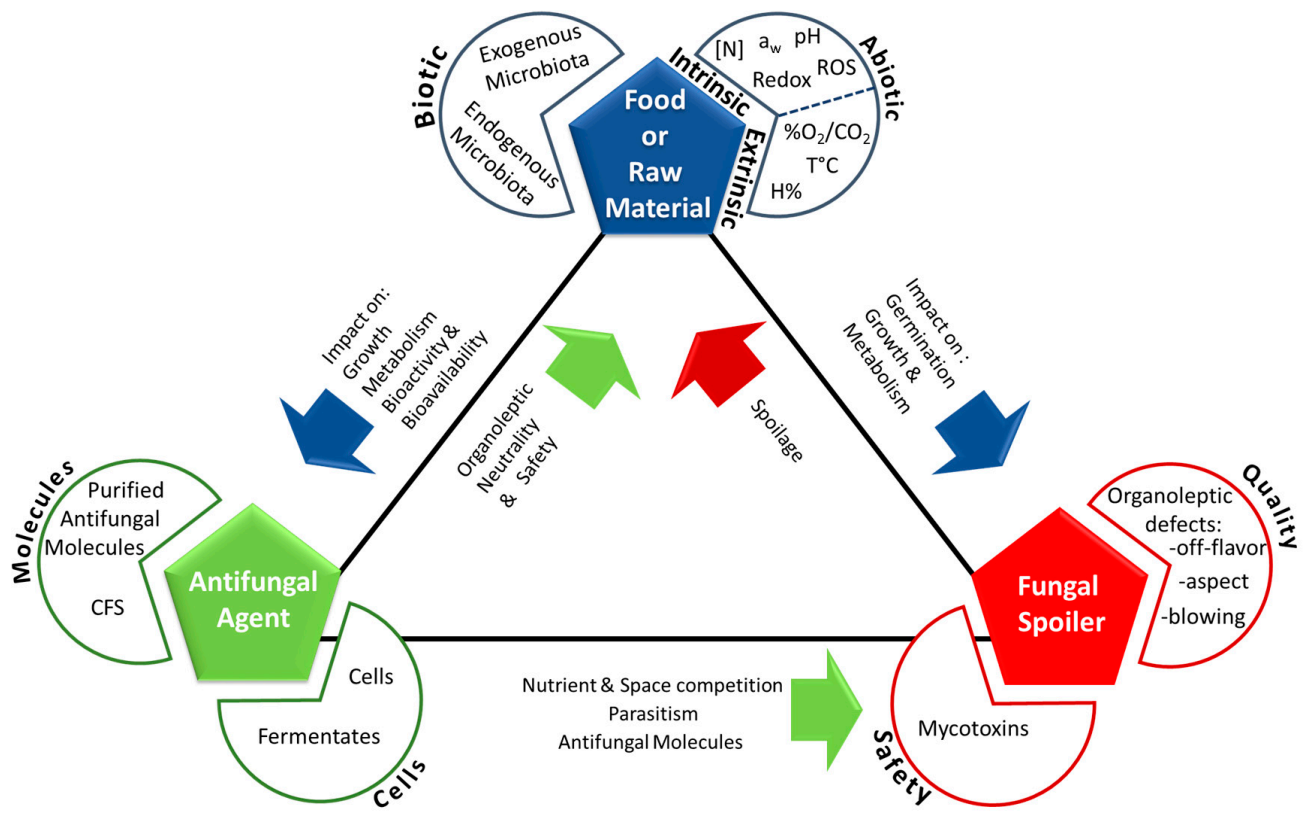

Figure 1. Diagram representing possible interactions occurring between food or raw material, antifungal agents, and fungal spoilers in a biopreservation context (CFS: culture-free supernatant, ROS: Reactive Oxygen Species, [N]: nutrient content, $\mathrm{H} \%$ : hygrometry).

Antifungal molecules can have (i) a target-specific mode of action, such as natamycin which blocks fungal growth via a specific interaction with ergosterol in the membrane, or (ii) a non-specific mode of action. In the latter case, molecules can generate an acid $(\mathrm{pH})$ and/or an osmotic stress, which can draw water from the cytoplasm, or correspond to chaotropic and hydrophobic stressors that can weaken or inhibit non-covalent interactions between macromolecular systems by reducing water [168]. Chaotropicity, like water activity and $\mathrm{pH}$, could be a key parameter in preservation.

\subsection{Action Mechanisms of Antifungal Lactic Acid Bacteria and Propionibacteria in Fermented Foods}

Antibiosis (e.g., production of antifungal molecules) and $\mathrm{pH}$ decrease are the main factors contributing to $\mathrm{LAB}$ and $\mathrm{PAB}$ antifungal activity in foods. Indeed, $\mathrm{LAB}$ and PAB produce lactic 
and propionic acids as main fermentation end-products, respectively. Moreover, most antifungal LAB, being facultative or strictly heterofermentative lactobacilli and pediococci, are also able to produce acetate from pentose (facultative or strictly heterofermentative LAB) and ethanol, or acetate (in the absence or presence of $\mathrm{O}_{2}$ or other electron acceptors, respectively) and $\mathrm{CO}_{2}$ from hexose (strictly heterofermentative LAB). On the other hand, propionibacteria also produce acetic and succinic acids as well as $\mathrm{CO}_{2}$ through the transcarboxylase cycle, in molar ratios depending on the substrate, environmental conditions, and strain [169]. These organic acids, synthesized at important levels in food (e.g., in $\mathrm{g} / \mathrm{L}$ or $\mathrm{g} / \mathrm{kg}$ order), possess antifungal activities, especially acetate and propionate, whose minimum inhibitory concentrations (MICs) are 30 to 100 times inferior to that of lactate depending on the fungal target. Moreover, acetate MIC is reduced in the presence of lactate, indicating that they can act in synergy. Nevertheless, the production of the aforementioned acids is not sufficient to explain antifungal activity of LAB and PAB [170].

For antifungal LAB, many molecules, produced at low quantities $(\mathrm{mg} / \mathrm{L}$ or $\mathrm{mg} / \mathrm{kg})$ and below their individual MIC, are also likely to act in synergy with lactic and acetic acids. The nature and quantity of these compounds is species- and strain-dependent and so far, a very large array of molecules has been described in the literature [24]. They include other organic acids (hydrocinnamic acid, DL- $\beta$-phenyllactic acid, DL- $\beta$-hydroxyphenyllactic acid, polyporic acid, azelaic acid, 2-hydroxybenzoic acid, 4-hydroxybenzoic acid, p-coumaric acid, vanillic acid, caffeic acid, succinic acid, 2-pyrrolidone-5-carboxylic acid), fatty acids (decanoic acid, 3-hydroxydecanoic acid, (S)-(-)-2-hydroxyisocapric acid, coriolic acid, ricinoleic acid), cyclopeptides [cyclo(L-Pro-L-Pro), cyclo(L-Leu-L-Pro), cyclo(L-Tyr-L-Pro), cyclo(L-Met-L-Pro), cyclo(Phe-Pro), cyclo(Phe-OH-Pro), cyclo(L-Phe-L-Pro), cyclo(L-Phe-trans-4- OH-L-Pro), cyclo(L-His-L-Pro), and cyclo(Leu-Leu)], reuterin, hydrogen peroxide, and volatile compounds such as diacetyl [101,171-178]. For example, Miezkin et al. (2017) [175] attributed the antifungal effect of L. harbinensis K.V9.3.1.Np to the synergistic action of acetic, lactic, 2-pyrrolidone-5-carboxylic, (S)-(-)-2-hydroxyisocapric, and 2-hydroxybenzoic acids, while Aunsbjerg et al. (2015) [101] showed that increased production of diacetyl and to a smaller extent, 2,3-pentadione, acetic acid, and butanoic acid were involved in the antifungal activity of L. paracasei DGCC 2132. In another study, Le Lay et al. (2016) [179] showed that lactic, acetic, and propionic acids, ethanol and hydrogen peroxide, as well as other compounds present at low levels such as DL- $\beta$-phenyllactic, DL- $\beta$-hydroxyphenyllactic, azelaic, and (S)-(-)-2-hydroxyisocapric acids were responsible for the antifungal activity of lactobacilli and propionibacteria CFS active against spoilage molds in bakery products. A fraction extracted from a culture of Lactococcus sp. BSN307, that contained 2,4-di-tert-butylphenol, was effective for wheat grains preservation from the attack by $A$. niger and different Fusarium sp. [110]

For propionibacteria, the four dairy species (P. freudenreichii, P. acidipropionici, P. thoenii, and P. jensenii) also produce DL- $\beta$-phenyllactic acid .[77] Propionicin PLG-1, a 9328 Da bacteriocin produced by $P$. thoenii P127, is active against various bacteria but also yeast and mold species [180]. Several antimicrobial organic acids acting in synergy have been identified in a protective mixed culture of P. jensenii SM11 and L. paracasei strain including 2-pyrrolidone-5-carboxylic, DL- $\beta$-phenyllactic, and DL- $\beta$-hydroxyphenyllactic acids [181]

The action mode of organic acids is quite well understood [182]. Organic acids, under their undissociated form, can diffuse through the microorganism membrane and dissociates in the cell, thereby causing a decrease in intracellular $\mathrm{pH}$. The accumulation of toxic ions combined with membrane disruption, inhibition of essential metabolic reactions, and/or stress in intracellular $\mathrm{pH}$ homeostasis may finally lead to cell death. In this so-called 'weak acid theory', the acid pKa value and the $\mathrm{pH}$ of the medium are therefore important factors influencing antifungal activity of organic acids. Indeed, the higher the $\mathrm{pKa}$ and the lower the medium $\mathrm{pH}$, the more the acid will be in its undissociated form and the higher will be its effect on intracellular $\mathrm{pH}$ and antifungal activity. However, not all organic acids act this way. For example, sorbic acid was shown to inhibit plasma-membrane $\mathrm{H}^{+}$-ATPase proton pump but not to decrease intracellular $\mathrm{pH}$ in S. cerevisiae [183]. It is also worth mentioning that 
phenolic compounds (4-hydroxy benzoic acid, vanillic acid); acetic, propanoic, and butanoic acids; acetoin; ethanol; and other volatile compounds can act as chaotrophic stressors $[168,184,185]$.

It should be highlighted that the multiplicity and variety of antifungal molecules identified so far in $\mathrm{LAB}$ or $\mathrm{PAB}$ render difficult their exhaustive identification and quantification. Therefore, in future studies, it would be desirable to conduct large targeted- and untargeted assays for antifungal molecule identification and quantification, by combining different extraction and analysis methods, such as LC-MS/MS or GC-MS. In this context, Brosnan et al. (2012, 2014) [186-188] developed two methods to extract and simultaneously detect and quantify a large variety of antifungal compounds. The method was further slightly modified and applied by Le Lay et al. (2016) [179]. Future work should also include the investigation of individual and combined effects of mixtures of antifungal molecules identified so far. Chaotrophicity could also be taken into account to determine the mechanism of action of antifungal microorganisms, nevertheless further studies must be done to determine the chaotrophicity of the mixture of identified molecules produced at low concentration by antifungal microorganisms of interest in food. Concerning their action mode against different fungal targets, only few studies looked at the effects of antifungal molecules produced by bioprotective LAB on fungal physiology in the food context. Crowley et al., (2013)[189] investigated the response of Aspergillus fumigatus Af293 (at the transcriptomic and morphological levels) to antifungal molecules present in L. plantarum 16 CFS. These authors observed an altered transcription of a large variety of cellular functions involved in metabolism, transport, signaling, ergosterol biosynthesis, and cell stress, including that of LaeA, a global regulator of secondary metabolism. More recently, Mieszkin et al. (2017) [175] investigated the action mechanisms involved in the bioprotective effect of L. harbinensis K.V9.3.1.Np against Y. lipolytica in fermented milk. The CFS obtained after milk fermentation by yogurt starters in co-culture with the bioprotective strain had a fungistatic rather than a fungicidal effect accompanied with a significant loss of cultivability in the studied yeast. It also led to membrane depolarization and intracellular $\mathrm{pH}$ decrease, as well as morphological changes including membrane collapsing and cell lysis.

\subsection{Action Mechanisms of Antifungal Yeasts and Molds in Fermented Foods}

As mentioned in the previous sections, selected yeasts and molds can be used as antifungal agents in dry-fermented foods to inhibit the growth of undesirable fungi. Their abilities to compete for space and nutrients with spoilage fungi as well as to produce antifungal molecules such as antifungal proteins and volatile compounds explain their antifungal properties [26]. Núñez et al. (2015) [136] showed that $D$. hansenii isolates are active against spoilage molds from dry-cured ham produced 2-methyl-1-butanol and other volatiles as well as unidentified diffusible molecules which were active against $P$. verrucosum. M. guilliermondi LCF1353, in combination with added L. plantarum 1 A7 and $W$. anomalus 1695 strains that were previously selected for their antifungal activity against $P$. roqueforti in wheat flour bread [92] produced ethyl acetate and a $\beta$-1,3-glucanase during dough fermentation, which were efficient in preventing fungal contamination of bread slices without negatively affecting bread sensorial properties [190]. As recently reviewed by Delgado et al. (2016a, 2016b) [26,191] molds can produce various peptides and proteins with antifungal properties, including basic, cysteine-rich antifungal proteins (AFPs) with a 5.5-10 kDa molecular weight. Their structure makes them highly stable to low $\mathrm{pH}$, heat, and proteolysis which is compatible with their possible antifungal role in fermented foods such as cheeses and fermented meats. After binding the cell wall or being internalized by target fungi, AFPs can alter chitin synthesis leading to cell death, or increase intracellular reactive oxygen species (ROS) levels, leading to cell permeabilization and apoptosis [26].

\subsection{Action Mechanisms of Antifungal Yeasts and Bacillus spp. for Control of Postharvest Diseases}

Biopreservation agents used for controlling postharvest disease have complex action mechanisms including competition for nutrients (e.g., iron and carbon sources) and space [71,130,192], antibiosis (e.g., production of antifungal molecules such as peptides and lipopeptides and volatile 
organic compounds) [24,101,193-195], mycoparasitism associated with lytic enzyme production (e.g., glucanases, chitinases, and proteases) [157,195] and induced host resistance [29].

For example, the group of cyclic lipopeptides (surfactins, fengycins, and iturins) produced by B. subtilis and other Bacillus sp. strains have been shown to protect host plants from a number of pathogens including fungi $[154,193]$. Cyclic lipopeptides possess surface properties that are believed to contribute to the ability of B. subtilis cells to spread and colonize surfaces, whereas the fengycin and iturin molecular families are strongly toxic for fungi $[154,196]$. New antifungal peptides are continuously characterized, as for example, a large peptide synthetized by Bacillus subtilis B25, which inhibits the growth of Fusarium oxysporum f. sp. cubense, a pathogen infecting bananas [197].

Volatile antifungal compounds that can act at a distance are produced by antifungal species, thus opening new possibilities to control food spoilage such as biofumigation, provided that the safety of this mode of application is ensured [198]. A variety of antifungal volatiles have been identified-including 2-phenylethanol, 2-methyl-1-butanol, 3-methyl-1-butanol, 2-methyl-1-propanol, 5-pentyl-2-furaldehyde, 2-nonanone, and 2-ethyl-1-hexanol [18], 2-Phenylethanol was identified for example as one of the antifungal volatiles produced by a strain of $P$. expansum R82 active against different species of postharvest fungal pathogens [199] and its mechanisms of action against $P$. digitatum and P. italicum investigated [200].

Many different mechanisms of action were identified in yeasts active against grape pathogens. The most important are the production of laminarinases, antifungal volatiles or growth-inhibiting metabolites, the inhibition of fungal spore germination, decrease of germinal tube length, and the competition for carbon sources and iron [201]. At least two of the mechanisms of action were present in the yeast isolates assayed, with many different 'antifungal patterns' found according to the isolates. In Pichia anomala strain WRL-076, used as a biocontrol agent to reduce aflatoxin contamination of tree nuts, 2-phenylethanol was identified as an antifungal volatile compound, which inhibited spore germination, growth, and aflatoxin production in A. flavus [202]

These aspects were recently reviewed in detail by Spadaro and Droby (2016) [18]. Di Francesco et al. (2016) [18] as well as Rahman (2017) [203]. Biofilm formation, quorum sensing, and oxidative stress were also underlined in these reviews as other important key factors in the action mode of these biocontrol agents. These reviews also pointed out the potential of omics techniques to study antagonist-pathogen-host interactions.

\section{Implementation of Biopreservation Methods against Fungal Spoilage}

\subsection{Optimization and Application Modes}

Once antifungal cultures have been selected and/or the molecules supporting the activity have been identified, the next questions that can be raised concern (i) the potential enhancement of their activity in terms of efficiency or action spectrum (i.e., the number of inhibited species/strains) and (ii) the antifungal product type and its application mode.

Concerning optimization of the antifungal activity, several methods have been developed to enhance the production of bioactive molecules or extend their action spectrum, and correspond to the use of microorganisms in co-cultures, addition of enhancing molecules as precursors to trigger their biosynthetic pathways, induction of stress conditions to cultures, or to associate microbial cultures with other active molecules. For example, mixed fungal cultures using Phomopsis sp. K38 and Alternaria sp. E33 [204] were shown to create a new cyclic tetrapeptide (cyclo-[L-leucyl-trans-4-hydroxy-L-prolyl-D-leucyl-trans-4-hydroxy-L-proline]), which was shown to be active against Gaeumannomyces graminis, Rhizoctonia cerealis, Helminthosporium sativum, and Fusarium graminearum in vitro. Co-cultures can also positively stimulate antifungal activity as observed with a kefir symbiotic consortium combining three bioprotective LAB species (L. plantarum, L. kefir, and Lactococcus lactis subsp. lactis) that was able to extend shelf life of a maize food product called 'arepa' against $A$. flavus contamination [113]. The use of an association of antifungal strains each 
exhibiting antifungal activity towards different fungal targets may be a way to enlarge the antifungal spectrum of action. Another means to enhance bioprotective activities is the addition of certain substrates in the medium or food product. Certain substrates can potentially inhibit antifungal microorganism development while others may enhance their activity. Glycerol is known to enhance the antifungal activity of LAB. For example, the use of immobilized cells of L. reuteri in a glycerol based medium enhanced reuterin production [102], a widely used preservative in foods. In the same way, Le Lay et al. [63] observed that the antifungal activity of L. brevis Lu35 and L. reuteri 5529 was increased on wheat based medium supplemented with $2.5 \%$ olive oil and $150 \mathrm{mM}$ glycerol, respectively. Other examples correspond to the use of sorbitol [205] or xylitol or galactosyl-xylitol [115] with Lactobacillus strains to inhibit a wide spectrum of fungi in vitro, while no inhibition was observed if other media were used. Then, stress conditions such as temperature, $\mathrm{pH}$, nutrient availability, and cell population density during culture of antifungal microorganism can trigger metabolic pathways that enable the bacterium to cope with the stressful environment [206] which leads to the production of diverse metabolites including antifungal molecules.

Finally, the association of bioprotective cultures with other molecules, such as chitosan, has been described and is currently used to protect postharvest crops by the coating technique. Chitosan protects fruits and vegetables from fungal decay, creates a barrier with the environment and delays ripening. A study by [129] tested the coating of citrus fruits with a chitosan mixture containing a P. membranifaciens culture to prevent $C$. gloeosporioides fungal infections which was more effective than using the yeast culture alone. Similarly, 2-hydroxybenzoic acid enhanced the biocontrol efficacy of the yeast R. glutinis against P. expansum and A. alternata in cherry fruit [207] Finally, the role of other ingredients in foods to help extend product shelf-life is also important. The use of spices combined with an antifungal LAB culture to efficiently prevent fungal spoilage of an Ethiopian spiced fermented cottage cheese was recently described [208].

Concerning the antifungal agent type, they correspond to the use of cells (as adjunct cultures), their fermentates, CFS, or partially or totally purified compounds, the best example being natamycin, a universal antifungal agent from Streptomyces natalensis. The type of antifungal agent combined with the targeted food matrix will then determine the application mode. Legislative aspects may also have to be taken into account. Application modes mainly correspond to the addition in the product as adjunct culture in fermented products or ingredient, spraying, dipping, or drenching. In bakery products, Le Lay et al. (2016) [63] used two different application modes for challenge tests. In milk bread rolls, a fermented product, cell cultures were added to the classical starters and showed that, for some strains, the antifungal activity was conserved despite the baking process. For pound cake, a non-fermented product, bioprotective action was performed through surface spraying. Also, L. amylovorus DSM19280 used as a starter in the preparation of gluten-free quinoa bread proved to extend the mold free shelf-life [80] The protection of post-harvest crops and cereals can be performed by dipping food in the active culture or cell suspension as shown by Pantelides et al. (2015) [70] with wounded grape berries and Perez et al. (2016) [131] with lemons, for biocontrol of B. cinerea and P. italicum, respectively. As for spraying, pulverization of lactic acid bacteria strain cell suspensions over wounded and non-wounded apples protected the fruits from P. expansum colonization [116].

\subsection{Constraints}

As previously stated, due to ever increasing societal demands for preservative-free foods, the use of antifungal cultures for biopreservation has triggered a strong interest in the scientific community. However, although numerous studies have been reported in the literature, only few commercial solutions are available to date (Table 2). This is due to several constraints that may impair the commercialization of candidate antifungal cultures. 
Table 2. Examples of bioprotective cultures and fermentates used in processed food and postharvest fruits available in the market.

\begin{tabular}{|c|c|c|c|c|}
\hline Product Name & Application Field & Properties & Composition & Manufacturer \\
\hline Holdbac YM-B or YM-C & fermented food and white cheeses & protection against yeasts and molds & $\begin{array}{l}\text { Lactobacillus rhamnosus and Propionibacterium } \\
\text { freudenreichii subsp. shermanii }\end{array}$ & DuPont Danisco \\
\hline Holdbac YM-XPM & fermented dairy and mild acidic yogurt & protection against yeasts and molds & Lactobacillus plantarum & DuPont Danisco \\
\hline Holdbac YM-XPK & all types of cheeses & protection against yeasts and molds & Lactobacillus plantarum & DuPont Danisco \\
\hline FreshQ 1 and FreshQ 4 & cottage cheese & protection against yeasts and molds & Lactobacillus rhamnosus and Lactobacillus paracasei & CHR Hansen \\
\hline FreshQ 2 & cottage cheese & protection against yeasts and molds & Lactobacillus rhamnosus & CHR Hansen \\
\hline FreshQ 5 & cottage cheese & protection against yeasts and molds & Lactobacillus paracasei & CHR Hansen \\
\hline Natamax & $\begin{array}{l}\text { fruit juices, wine, surface of dry-ripened food, } \\
\text { dairy, and bakery products }\end{array}$ & protection against yeasts and molds & Natamycin produced by Streptomyces natalensis & DuPont Danisco \\
\hline MicroGard & $\begin{array}{l}\text { sauces, salad dressings, prepared meals, cured } \\
\text { meat, pastas, bakery and dairy products, hash } \\
\text { brown potatoes }\end{array}$ & $\begin{array}{l}\text { protection against Gram-positive bacteria, } \\
\text { Gram-negative bacteria, yeasts, and molds }\end{array}$ & $\begin{array}{l}\text { Fermentate (skim milk or dextrose) of } \\
\text { Propionibacterium freudenreichii subsp. shermani }\end{array}$ & DuPont Danisco \\
\hline Hi Shield P & $\begin{array}{l}\text { bakery products, salad dressings, and general } \\
\text { used in food industry }\end{array}$ & $\begin{array}{l}\text { protection against molds, yeasts (Pichia anomala), and } \\
\text { bacteria (Bacillus subtilis); increase sour taste; and reduce } \\
\text { salt content (flavor improver and enhancer) }\end{array}$ & Fermentate (corn) of lactic acid bacteria and yeasts & HI-FOOD S.p.A. \\
\hline $\begin{array}{l}\text { Inhibit FOG, Inhibit 2800, } \\
\text { Inhibit 1900CW, Inhibit } 3600 \\
\text { and Inhibit 2100NF }\end{array}$ & $\begin{array}{l}\text { bakery products, cheeses, meats, salad dressings, } \\
\text { condiments, dips, spreads, and meats }\end{array}$ & $\begin{array}{l}\text { protection against molds, yeasts, } \\
\text { and Gram-negative bacteria }\end{array}$ & $\begin{array}{l}\text { Fermentates (dextrose, wheat, wheat flour, whey, } \\
\text { brown rice) of Propionibacterium freudenreichii }\end{array}$ & Mezzoni Foods \\
\hline Biosafe 10LP & cherries, pome fruits, citrus, and potatoes & $\begin{array}{l}\text { protection against Penicillium expansum, Botrytis cinerea, } \\
\text { Mucor piriformis, Fusarium sambucinum, Helminthosporium } \\
\text { solani, and Rhizopus stolonifer }\end{array}$ & Pseudomonas syringae & Nu Farm Inc. USA \\
\hline Aspire & citrus and pome fruit & protection against molds (P. expansum and Botrytis cinerea) & Candida oleophila & Ecogen Inc. USA \\
\hline Befresh & fresh fermented milk products & control the growth of yeast and molds & $\begin{array}{l}\text { Lactobacillus paracasei and Propionibacterium } \\
\text { freudenreichii subsp. shermanii, }\end{array}$ & Handary \\
\hline Candifruit & pome fruit & $\begin{array}{l}\text { protection against Botrytis cinerea, Penicillium expansum, } \\
\text { and Rhizopus stolonifer }\end{array}$ & Candida sake & $\begin{array}{l}\text { Sipcam-Inaagri, SA } \\
\text { (Valencia, Spain) }\end{array}$ \\
\hline Boni-Protect & pome fruit & $\begin{array}{l}\text { protection against Botrytis cinerea, Monilinia fructigena, } \\
\text { Penicillium expansum, and Pezicula malicortici }\end{array}$ & Aureobasidium pullulan & BioFerm GMbH, Germany \\
\hline Shemer & citrus fruit, stone fruits, and berries & $\begin{array}{l}\text { protection against Aspergillus niger, Botrytis cinerea, } \\
\text { Penicillium expansum, Penicillium digitatum, Penicillium } \\
\text { italicum, and Rhizopus stolonifer }\end{array}$ & Metschnikowia fructicola & Bayer Cropscience, Israel \\
\hline Pantovital & citrus and pome fruit & $\begin{array}{l}\text { protection against Botrytis cinerea, Penicillium expansum, } \\
\text { Penicillium digitatum, Penicillium italicum, } \\
\text { and Rhizopus stolonifer }\end{array}$ & Pantoea agglomerans & BioDURCAL S.L. \\
\hline YieldPlus & citrus, apple, and pear fruit & not detailed & Cryptococcus albidus & $\begin{array}{l}\text { Anchor Bio-Technologies, } \\
\text { Cape Town, South Africa }\end{array}$ \\
\hline Nexy & pome fruit & protection against Botrytis cinerea and Penicillium expansum & Candida oleophila & BioNext sprl, France \\
\hline Serenade & grapes, legumes, pome fruits, and peanuts & $\begin{array}{l}\text { protection against fungi causing powdery mildew, late } \\
\text { blight brown rot, fireblight }\end{array}$ & Bacillus subtilis & Agra Quees Inc. \\
\hline
\end{tabular}


The first constraint concerns the gap between an observed activity in a culture medium (the in vitro effect) and the actual activity in the final matrix (in situ effect). Several studies have described that numerous microorganisms can show interesting results in in vitro screening conditions but that the actual number drastically decreases when tested in the target food matrices. For example, Delavenne et al. (2013) [78] tested 11 bacteria exhibiting important antifungal activity in vitro and only 1 strain. belonging to the L. harbinensis species, proved to be active in yogurt. One way to get around this problem is to use semi-synthetic media that can closely mimic food composition. However, as shown by Le Lay et al. (2016) [63] the use of a wheat based medium, although more effective than MRS for antifungal activity expression, did not completely represent the complexity of bakery products and therefore, the number of efficient antifungal strains was still far smaller when tested on sweet bread and pound cake. The observed differences are obviously linked to the complexity of the food or raw material matrices. They are characterized by different abiotic and biotic factors that can impact the growth and metabolism of the antifungal cultures or the bioavailability and bioactivity of antifungal compounds. The fungal spoiler load, the antifungal agent load or concentration, and the treatment moment will also impact efficiency. The interactions between fungal spoilers, food matrices, and antifungal agents are illustrated in Figure 1. Therefore, it is crucial to test the actual antifungal efficiency of potential bioprotective strains on the final product intended for use (challenge-tests).

Direct testing on the food matrix also enables the study of another important constraint linked to the direct addition of bacteria into a given food matrix. Indeed, this method directly provides information related to its impact on the organoleptic qualities of the product. According to its metabolism, an antifungal culture may exert a positive, neutral, or negative impact. Some examples are as follows, heterofermentative antifungal microorganisms may lead to $\mathrm{CO}_{2}$ production directly affecting the product's aspect (bubble or air-pocket formation) or the packaging (blown packages), while other microbial candidates may exhibit intense enzymatic activities (i.e., proteolytic, amylolytic, or lipolytic) directly affecting product texture and overall aspect. Finally, other antifungal strains may produce aroma compounds leading to off-flavor defects. In this context, antifungal culture selection should aim towards organoleptic neutrality. This can be achieved by the use of sensory evaluation methods (i.e., triangle test, rapid methods for sensory profiling) to define and quantify a potential organoleptic impact in the final product. Notably, the quantity of antifungal agent to be added for efficient activity may be incompatible from an organoleptic but also a cost point of view.

The safety of the selected cultures or their CFS is another key aspect beyond these first two constraints linked to antifungal culture efficiencies and product quality issues. Safety assessment, including any regulatory considerations that need to be addressed, has now become a key step during strain selection for biotechnological use in general. In this sense, the European Union developed the Qualified Presumption of Safety (QPS) approach as a means to assess the safety of a broad range of biological agents with intended uses as sources of food and feed additives, enzymes, and plant protection products [209] and can more generally be considered as a premarket evaluation of microorganisms used in food and feed production [210] to ensure their safe use. In the United States, a similar 'Generally Recognized as Safe' (GRAS) status exists for food and substances used in food [211]. This status implies that a given GRAS substance has been efficiently shown to be safe under the conditions intended for its use. In both cases, regularly updated lists of QPS recommended biological agents or GRAS food substances are provided by the corresponding regulatory bodies (EFSA and FDA, respectively). In the case of bioprotective cultures, fermentates, or CFS, the goal is to intentionally and safely add them to a food matrix for their bioprotective properties while ensuring consumer safety. In this context, it is necessary to follow a rigorous safety assessment procedure, taking multiple criteria into account and a model has recently been proposed for LAB and propionibacteria safety evaluation by Coton et al. [212]. First of all, according to the strain intended for direct use or the strain used to produce the fermentates/CFS, the following criteria may be considered (i) overall body of knowledge including history and intended end use; (ii) well-defined taxonomy with identification at the species level; and (iii) any potential safety hazards including pathogenicity factors, unwanted 
antibiotic resistance profiles (especially acquired resistances via horizontal gene transfer events that can potentially lead to further dissemination of this trait), and undesirable compound formation including biogenic amines, allergens, or toxins [209-215]. In the case of the most commonly considered bacterial groups for industrial applications (i.e., LAB and propionibacteria), many members of these groups already have a long history of safe use in foods and/or may already have a GRAS or QPS status. For such cultures, the safety criteria that need to be included during evaluation can be reduced to the current body of knowledge and intended end use, well-defined taxonomy (although in some cases, this may include very recently described species), antibiotic resistance profiles, and biogenic amine production (no known toxins or virulence factors have been described so far). Biogenic amines produced by LAB species in fermented foods, including dairy products, have been well reviewed in the literature (see [216-218]). Their production has been described as a strain-dependent trait for many species $[219,220]$ which emphasizes the need to include this feature during safety assessment. By following this safety assessment procedure, a given culture or corresponding fermentate/CFS intended to be used as a bioprotective agent may be excluded for safe use if multi-resistant antibiotic profiles are identified (in particular, any acquired resistances and/or the presence of the corresponding biosynthesis genes and mobile elements in the genome) or if any undesirable compounds such as biogenic amines (especially tyramine, histamine, putrescine, or cadaverine) potentially known to have a negative physiological effect on sensitive consumers [218,221,222] and/or if the corresponding biosynthesis genes are identified. In the case of other bacterial groups (i.e., Bacillus) or fungal strains (yeasts or molds), safety assessment should also include determining whether the strain produces other undesirable compounds (especially toxins by bacteria or mycotoxins by molds). If detected, this would exclude their use in industrial applications.

Other constraints / criteria that must be considered before marketing a candidate bioprotective culture-or its corresponding fermentate/CFS - are related to strain selection, propagation, and preparation (i.e., selling format). As mentioned above, only few commercially available bioprotective cultures are on the market and this is likely due to the fact that not all criteria have been thoroughly studied or satisfied. Indeed, multiple requirements must be met, on top of the constraints mentioned above, knowing that any given bioprotective agent will be intentionally added to the food matrix at different steps during food processing and bioprotective activities must be conserved during shelf life. Preliminary tests to evaluate propagation to high population, microbial stability, and cell viability after preparation (in the selling format) and addition into the food matrix are required and bioprotective activity must also be maintained during the storage period $[29,87,223,224]$. This is an essential step as stability and/or activity may be altered according to how the protective culture is prepared. Different techniques can be used to prepare dry or liquid formulations, such as freeze-drying or spray drying techniques to produce active dry powder and metabolite(s) purification procedures. Dry formulations have been shown to be more advantageous for many reasons including longer shelf life, easier storage under non-refrigerated conditions, and ease of distribution [223,225]. In all cases, the impact of preparation procedures on the efficiency of protective cultures/fermentates/CFS should be determined. The most important criteria is to ensure that adequate shelf life is retained to ensure efficient use as a bioprotective agent in the food matrix. Notably, previous studies have shown that environmental conditions encountered in foods can have either positive or negative effects on bacterial antimicrobial activities [226-230] which again emphasizes the need for thorough in situ evaluation of candidate protective cultures before commercial use.

Finally, another constraint can be at the regulation level as, in some countries, the regulation definition of these antifungal agents (starter, ingredient, technological auxiliary, or even additive) can be raised. However, as discussed previously, the main antifungal agents correspond to microorganisms (or product of their metabolism) with a long history of use and GRAS or QPS status, thus guarantying an expected safety of use, provided that all mentioned safety aspects have been covered. 


\section{Conclusions}

The increasing societal demand for less processed and more natural food products-while conserving those products' quality, safety, and shelf-life-has raised the question of chemical preservative replacement. In this context, bacteria and fungi as well as their metabolites are natural alternatives of interest for use in food as bioprotective tools to fight fungal spoilage and to answer consumer demands and legislation. From an applied point of view, the difference between the number of studies and the number of available microbial cultures indicates that efforts are needed to facilitate their application in food commodities. One of the main aspects concerns the crucial role of in situ studies using adapted fungal targets during antifungal activity screening or confirmation processes. Also, safety assessment, organoleptic neutrality, and activity stability of the bioprotective cultures need to be evaluated prior to marketing. From a cognitive point of view, while antifungal compounds have been widely studied, and have generally been shown to act synergistically, there is still a lack of knowledge concerning the overall picture as to what molecules are involved and their action mechanism(s). The combination of pertinent biochemical analytic tools and omics methods should enable us to decipher antifungal action mechanisms, potentially identifying new levers for antifungal activity. Finally, if finding natural antifungal agents is a key factor, it can only be considered in good practices and within the HACCP context as one of the hurdle technologies to prevent fungal spoilage.

Conflicts of Interest: The authors declare no conflict of interest.

\section{References}

1. Gustavsson, J.; Cederberg, C.; Sonesson, U. Global Food Losses and Food Waste: Extent, Causes and Prevention; Food and Agriculture Organization of the United Nations: Rome, Italy, 2011; ISBN 978-92-5-107205-9.

2. FAO. Save Food: Global Initiative on Food Loss and Waste Reduction-Key Findings. Available online: http:/ / www.fao.org/save-food/resources/keyfindings/en/ (accessed on 2 May 2017).

3. Kitinoja, L.; Saran, S.; Roy, S.K.; Kader, A.A. Postharvest technology for developing countries: Challenges and opportunities in research, outreach and advocacy. J. Sci. Food Agric. 2011, 91, 597-603. [CrossRef] [PubMed]

4. Pitt, J.I.; Hocking, A.D. Fungi and Food Spoilage; Springer: Boston, MA, USA, 2009; ISBN 978-0-387-92206-5.

5. Sanzani, S.M.; Reverberi, M.; Geisen, R. Mycotoxins in harvested fruits and vegetables: Insights in producing fungi, biological role, conducive conditions, and tools to manage postharvest contamination. Postharvest Biol. Technol. 2016, 122, 95-105. [CrossRef]

6. Krupinsky, J.M.; Bailey, K.L.; McMullen, M.P.; Gossen, B.D.; Turkington, T.K. Managing plant disease risk in diversified cropping systems. Agron. J. 2002, 94, 198-209. [CrossRef]

7. Rojas-Graü, M.A.; Oms-Oliu, G.; Soliva-Fortuny, R.; Martín-Belloso, O. The use of packaging techniques to maintain freshness in fresh-cut fruits and vegetables: A review. Int. J. Food Sci. Technol. 2009, 44, 875-889. [CrossRef]

8. Verma, L.R.; Joshi, D.V.K. Postharvest Technology of Fruits and Vegetables: General Concepts and Principles; Indus Publishing: Lahore, Pakistan, 2000; ISBN 978-81-7387-108-5.

9. Dijksterhuis, J.; Houbraken, J.; Samson, R.A. 2 Fungal spoilage of crops and food. In Agricultural Applications; Kempken, F., Ed.; Springer: Berlin/Heidelberg, Germany, 2013; pp. 35-56, ISBN 978-3-642-36821-9.

10. Fellows, P.J. Food Processing Technology: Principles and Practice, 3rd ed.; Woodhead Publishing: Oxford, UK; Boca Raton, FL, USA, 2009; ISBN 978-1-84569-216-2.

11. Silva, M.; Lidon, F. Food preservatives-An overview on applications and side effects. Emir. J. Food Agric. 2016, 1. [CrossRef]

12. Stark, J.; Tan, H.S. Natamycin. In Food Preservatives; Springer: New York, NY, USA, 2003; pp. $179-195$.

13. Sharma, A.; Diwevidi, V.D.; Singh, S.; Pawar, K.K.; Jerman, M.; Singh, L.B.; Singh, S.; Srivastawav, D. Biological control and its important in agriculture. Int. J. Biotechnol. Bioeng. Res. 2013, 4, 175-180.

14. Oliveira, P.M.; Zannini, E.; Arendt, E.K. Cereal fungal infection, mycotoxins, and lactic acid bacteria mediated bioprotection: From crop farming to cereal products. Food Microbiol. 2014, 37, 78-95. [CrossRef] [PubMed] 
15. Kabaluk, J.T.; Svircev, A.M.; Goettel, M.S.; Woo, S.G. The Use and Regulation of Microbial Pesticides in Representative Jurisdictions Worldwide. Available online: http:/ /www.iobc-global.org/download/ Microbial_Regulation_Book_Kabaluk_et_al_2010.pdf (accessed on August 2010).

16. Lacroix, C. Protective Cultures, Antimicrobial Metabolites and Bacteriophages for Food and Beverage Biopreservation; Elsevier: Amsterdam, The Netherlands, 2010; ISBN 0-85709-052-6.

17. Robinson-Boyer, L.; Jeger, M.J.; Xu, X.-M.; Jeffries, P. Management of strawberry grey mould using mixtures of biocontrol agents with different mechanisms of action. Biocontrol Sci. Technol. 2009, 19, 1051-1065. [CrossRef]

18. Spadaro, D.; Droby, S. Development of biocontrol products for postharvest diseases of fruit: The importance of elucidating the mechanisms of action of yeast antagonists. Trends Food Sci. Technol. 2016, 47, 39-49. [CrossRef]

19. Deising, H.B.; Reimann, S.; Pascholati, S.F. Mechanisms and significance of fungicide resistance. Braz. J. Microbiol. 2008, 39, 286-295. [CrossRef] [PubMed]

20. Piper, P.W. Resistance of yeasts to weak organic acid food Preservatives. In Advances in Applied Microbiology; Elsevier: Amsterdam, The Netherlands, 2011; Volume 77, pp. 97-113, ISBN 978-0-12-387044-5.

21. Atarés, L.; Chiralt, A. Essential oils as additives in biodegradable films and coatings for active food packaging. Trends Food Sci. Technol. 2016, 48, 51-62. [CrossRef]

22. Pandey, A.K.; Kumar, P.; Singh, P.; Tripathi, N.N.; Bajpai, V.K. Essential Oils: Sources of Antimicrobials and Food Preservatives. Front. Microbiol. 2016, 7, 2161. [CrossRef] [PubMed]

23. Reyes-Jurado, F.; Franco-Vega, A.; Ramirez-Corona, N.; Palou, E.; Lopez-Malo, A. Essential Oils: Antimicrobial Activities, Extraction Methods, and Their Modeling. Food Eng. Rev. 2015, 7, 275-297. [CrossRef]

24. Crowley, S.; Mahony, J.; van Sinderen, D. Current perspectives on antifungal lactic acid bacteria as natural bio-preservatives. Trends Food Sci. Technol. 2013, 33, 93-109. [CrossRef]

25. Dalié, D.K.D.; Deschamps, A.M.; Richard-Forget, F. Lactic acid bacteria-Potential for control of mould growth and mycotoxins: A review. Food Control 2010, 21, 370-380. [CrossRef]

26. Delgado, J.; Owens, R.A.; Doyle, S.; Asensio, M.A.; Núñez, F. Manuscript title: Antifungal proteins from moulds: Analytical tools and potential application to dry-ripened foods. Appl. Microbiol. Biotechnol. 2016, 100, 6991-7000. [CrossRef] [PubMed]

27. Elsser-Gravesen, D.; Elsser-Gravesen, A. Biopreservatives. In Biotechnology of Food and Feed Additives; Zorn, H., Czermak, P., Eds.; Springer: Berlin/Heidelberg, Germany, 2013; Volume 143, pp. 29-49, ISBN 978-3-662-43760-5.

28. Leroy, F.; De Vuyst, L. Lactic acid bacteria as functional starter cultures for the food fermentation industry. Trends Food Sci. Technol. 2004, 15, 67-78. [CrossRef]

29. Liu, J.; Sui, Y.; Wisniewski, M.; Droby, S.; Liu, Y. Review: Utilization of antagonistic yeasts to manage postharvest fungal diseases of fruit. Int. J. Food Microbiol. 2013, 167, 153-160. [CrossRef] [PubMed]

30. Stiles, M.E. Biopreservation by lactic acid bacteria. Antonie Van Leeuwenhoek 1996, 70, 331-345. [CrossRef] [PubMed]

31. Sharma, R.R.; Singh, D.; Singh, R. Biological control of postharvest diseases of fruits and vegetables by microbial antagonists: A review. Biol. Control 2009, 50, 205-221. [CrossRef]

32. Punja, Z.K.; Utkhede, R.S. Using fungi and yeasts to manage vegetable crop diseases. Trends Biotechnol. 2003, 21, 400-407. [CrossRef]

33. Gajbhiye, M.H.; Kapadnis, B.P. Antifungal-activity-producing lactic acid bacteria as biocontrol agents in plants. Biocontrol Sci. Technol. 2016, 26, 1451-1470. [CrossRef]

34. Nguyen, P.-A.; Strub, C.; Fontana, A.; Schorr-Galindo, S. Crop molds and mycotoxins: Alternative management using biocontrol. Biol. Control 2017, 104, 10-27. [CrossRef]

35. Buron, N.; Coton, M.; Legendre, P.; Ledauphin, J.; Kientz-Bouchart, V.; Guichard, H.; Barillier, D.; Coton, E. Implications of Lactobacillus collinoides and Brettanomyces/Dekkera anomala in phenolic off-flavour defects of ciders. Int. J. Food Microbiol. 2012, 153, 159-165. [CrossRef] [PubMed]

36. Romano, A.; Perello, M.C.; de Revel, G.; Lonvaud-Funel, A. Growth and volatile compound production by Brettanomyces/Dekkera bruxellensis in red wine. J. Appl. Microbiol. 2008, 104, 1577-1585. [CrossRef] [PubMed]

37. La Guerche, S.; Dauphin, B.; Pons, M.; Blancard, D.; Darriet, P. Characterization of Some Mushroom and Earthy Off-Odors Microbially Induced by the Development of Rot on Grapes. J. Agric. Food Chem. 2006, 54, 9193-9200. [CrossRef] [PubMed] 
38. Rousseaux, S.; Diguta, C.F.; Radoï-Matei, F.; Alexandre, H.; Guilloux-Bénatier, M. Non-Botrytis grape-rotting fungi responsible for earthy and moldy off-flavors and mycotoxins. Food Microbiol. 2014, 38, 104-121. [CrossRef] [PubMed]

39. Kabisch, J.; Erl-Höning, C.; Wenning, M.; Böhnlein, C.; Gareis, M.; Pichner, R. Spoilage of vacuum-packed beef by the yeast Kazachstania psychrophila. Food Microbiol. 2016, 53, 15-23. [CrossRef] [PubMed]

40. Spanoghe, M.; Godoy Jara, M.; Rivière, J.; Lanterbecq, D.; Gadenne, M.; Marique, T. Development and application of a quantitative real-time PCR assay for rapid detection of the multifaceted yeast Kazachstania servazzii in food. Food Microbiol. 2017, 62, 133-140. [CrossRef] [PubMed]

41. Wrent, P.; Rivas, E.-M.; de Prado, E.; Peinado, J.; de Silóniz, M.-I. Assessment of the Factors Contributing to the Growth or Spoilage of Meyerozyma guilliermondii in Organic Yogurt: Comparison of Methods for Strain Differentiation. Microorganisms 2015, 3, 428-440. [CrossRef] [PubMed]

42. Groenewald, M.; Boekhout, T.; Neuvéglise, C.; Gaillardin, C.; van Dijck, P.W.M.; Wyss, M. Yarrowia lipolytica: Safety assessment of an oleaginous yeast with a great industrial potential. Crit. Rev. Microbiol. 2014, 40, 187-206. [CrossRef] [PubMed]

43. Park, D.L.; Njapau, H.; Boutrif, E. Minimizing risks posed by mycotoxins utilizing the HACCP concept. Food Nutr. Agric. 1999, 23, 49-54.

44. Sellamani, M.; Kalagatur, N.K.; Siddaiah, C.; Mudili, V.; Krishna, K.; Natarajan, G.; Putcha, V.L.R. Antifungal and Zearalenone Inhibitory Activity of Pediococcus pentosaceus Isolated from Dairy Products on Fusarium graminearum. Front. Microbiol. 2016, 7, 890. [CrossRef] [PubMed]

45. Van Egmond, H.P.; Schothorst, R.C.; Jonker, M.A. Regulations relating to mycotoxins in food. Anal. Bioanal. Chem. 2007, 389, 147-157. [CrossRef] [PubMed]

46. Ostry, V. Alternaria mycotoxins: An overview of chemical characterization, producers, toxicity, analysis and occurrence in foodstuffs. World Mycotoxin J. 2008, 1, 175-188. [CrossRef]

47. Smith, M.-C.; Madec, S.; Coton, E.; Hymery, N. Natural Co-Occurrence of Mycotoxins in Foods and Feeds and Their in Vitro Combined Toxicological Effects. Toxins 2016, 8, 94. [CrossRef] [PubMed]

48. Fleet, G.H. Yeasts in foods and beverages: Impact on product quality and safety. Curr. Opin. Biotechnol. 2007, 18, 170-175. [CrossRef] [PubMed]

49. Garnier, L.; Valence, F.; Pawtowski, A.; Auhustsinava-Galerne, L.; Frotté, N.; Baroncelli, R.; Deniel, F.; Coton, E.; Mounier, J. Diversity of spoilage fungi associated with various French dairy products. Int. J. Food Microbiol. 2016. [CrossRef] [PubMed]

50. Lozano-Ojalvo, D.; Rodríguez, A.; Cordero, M.; Bernáldez, V.; Reyes-Prieto, M.; Córdoba, J.J. Characterisation and detection of spoilage mould responsible for black spot in dry-cured fermented sausages. Meat Sci. 2015, 100, 283-290. [CrossRef] [PubMed]

51. Nilsson, R.H.; Kristiansson, E.; Ryberg, M.; Hallenberg, N.; Larsson, K.H. Intraspecific ITS variability in the kingdom Fungi as expressed in the international sequence databases and its implications for molecular species identification. Evol. Bioinform. 2008, 4, 193-201.

52. Sholberg, P.L.; Conway, W.S. Postharvest pathology. In The Commercial Storage of Fruits, Vegetables, and Florist and Nursery Stocks, USDA-ARS Agriculture Handbook; U.S. Department of Agriculture, Agricultural Research Service: Washington, DC, USA, 2004.

53. Gill, C.O.; Lowry, P.D.; Di Menna, M.E. A Note on the Identities of Organisms Causing Black Spot Spoilage of Meat. J. Appl. Bacteriol. 1981, 51, 183-187. [CrossRef]

54. Rawat, S. Food Spoilage: Microorganisms and their prevention. Asian J. Plant Sci. Res. 2015, 5, 47-56.

55. Stevenson, A.; Cray, J.A.; Williams, J.P.; Santos, R.; Sahay, R.; Neuenkirchen, N.; McClure, C.D.; Grant, I.R.; Houghton, J.D.; Quinn, J.P. Others is there a common water-activity limit for the three domains of life? ISME J. 2015, 9, 1333-1351. [CrossRef] [PubMed]

56. Wolter, H.; Laing, E.; Viljoen, B.C. Isolation and identification of yeasts associated with intermediate moisture meats. Food Technol. Biotechnol. 2000, 38, 69-76.

57. Dakal, T.C.; Solieri, L.; Giudici, P. Adaptive response and tolerance to sugar and salt stress in the food yeast Zygosaccharomyces rouxii. Int. J. Food Microbiol. 2014, 185, 140-157. [CrossRef] [PubMed]

58. Leong, S.L.; Pettersson, O.V.; Rice, T.; Hocking, A.D.; Schnürer, J. The extreme xerophilic mould Xeromyces bisporus-Growth and competition at various water activities. Int. J. Food Microbiol. 2011, 145, 57-63. [CrossRef] [PubMed] 
59. Wheeler, K.A.; Hocking, A.D.; Pitt, J.I.; Anggawati, A.M. Fungi associated with Indonesian dried fish. Food Microbiol. 1986, 3, 351-357. [CrossRef]

60. Stevenson, A.; Hamill, P.G.; O’Kane, C.J.; Kminek, G.; Rummel, J.D.; Voytek, M.A.; Dijksterhuis, J.; Hallsworth, J.E. Aspergillus penicillioides differentiation and cell division at 0.585 water activity: Fungal cell division at 0.585 water activity. Environ. Microbiol. 2017, 19, 687-697. [CrossRef] [PubMed]

61. Zimmermann, M.; Miorelli, S.; Massaguer, P.R.; Aragão, G.M.F. Growth of Byssochlamys nivea in pineapple juice under the effect of water activity and ascospore age. Braz. J. Microbiol. 2011, 42, 203-210. [CrossRef] [PubMed]

62. Dos Santos, J.L.P.; Bernardi, A.O.; Pozza Morassi, L.L.; Silva, B.S.; Copetti, M.V.; Sant'Ana, A.S. Incidence, populations and diversity of fungi from raw materials, final products and air of processing environment of multigrain whole meal bread. Food Res. Int. 2016, 87, 103-108. [CrossRef]

63. Le Lay, C.; Mounier, J.; Vasseur, V.; Weill, A.; Le Blay, G.; Barbier, G.; Coton, E. In vitro and in situ screening of lactic acid bacteria and propionibacteria antifungal activities against bakery product spoilage molds. Food Control 2016, 60, 247-255. [CrossRef]

64. Hocking, A.D. Spoilage Problems: Problems caused by fungi. In Encyclopedia of Food Microbiology; Elsevier: Amsterdam, The Netherlands, 2014; pp. 471-481, ISBN 978-0-12-384733-1.

65. Gaggia, F.; Di Gioia, D.; Baffoni, L.; Biavati, B. The role of protective and probiotic cultures in food and feed and their impact in food safety. Trends Food Sci. Technol. 2011, 22, S58-S66. [CrossRef]

66. Wang, Y.-T.; Xue, Y.-R.; Liu, C.-H. A Brief Review of Bioactive Metabolites Derived from Deep-Sea Fungi. Mar. Drugs 2015, 13, 4594-4616. [CrossRef] [PubMed]

67. Vero, S.; Garmendia, G.; González, M.B.; Bentancur, O.; Wisniewski, M. Evaluation of yeasts obtained from Antarctic soil samples as biocontrol agents for the management of postharvest diseases of apple (Malus $\times$ domestica). FEMS Yeast Res. 2013, 13, 189-199. [CrossRef] [PubMed]

68. Cortés-Zavaleta, O.; López-Malo, A.; Hernández-Mendoza, A.; García, H.S. Antifungal activity of lactobacilli and its relationship with 3-phenyllactic acid production. Int. J. Food Microbiol. 2014, 173, 30-35. [CrossRef] [PubMed]

69. Russo, P.; Arena, M.P.; Fiocco, D.; Capozzi, V.; Drider, D.; Spano, G. Lactobacillus plantarum with broad antifungal activity: A promising approach to increase safety and shelf-life of cereal-based products. Int. J. Food Microbiol. 2017, 247, 48-54. [CrossRef] [PubMed]

70. Pantelides, I.S.; Christou, O.; Tsolakidou, M.-D.; Tsaltas, D.; Ioannou, N. Isolation, identification and in vitro screening of grapevine yeasts for the control of black aspergilli on grapes. Biol. Control 2015, 88, 46-53. [CrossRef]

71. Parafati, L.; Vitale, A.; Restuccia, C.; Cirvilleri, G. Biocontrol ability and action mechanism of food-isolated yeast strains against Botrytis cinerea causing post-harvest bunch rot of table grape. Food Microbiol. 2015, 47, 85-92. [CrossRef] [PubMed]

72. Ibrahim, M.; Kaushik, N.; Sowemimo, A.; Chhipa, H.; Koekemoer, T.; van de Venter, M.; Odukoya, O.A. Antifungal and antiproliferative activities of endophytic fungi isolated from the leaves of Markhamia tomentosa. Pharm. Biol. 2017, 55, 590-595. [CrossRef] [PubMed]

73. Tokpah, D.P.; Li, H.; Wang, L.; Liu, X.; Mulbah, Q.S.; Liu, H. An assessment system for screening effective bacteria as biological control agents against Magnaporthe grisea on rice. Biol. Control 2016, 103, 21-29. [CrossRef]

74. Inglin, R.C.; Stevens, M.J.A.; Meile, L.; Lacroix, C.; Meile, L. High-throughput screening assays for antibacterial and antifungal activities of Lactobacillus species. J. Microbiol. Methods 2015, 114, 26-29. [CrossRef] [PubMed]

75. Delavenne, E.; Mounier, J.; Déniel, F.; Barbier, G.; Le Blay, G. Biodiversity of antifungal lactic acid bacteria isolated from raw milk samples from cow, ewe and goat over one-year period. Int. J. Food Microbiol. 2012, 155, 185-190. [CrossRef] [PubMed]

76. Stiles, J.; Penkar, S.; Plocková, M.; Chumchalová, J.; Bullerman, L.B. Antifungal Activity of Sodium Acetate and Lactobacillus rhamnosus. J. Food Prot. 2002, 65, 1188-1191. [CrossRef] [PubMed]

77. Lind, H.; Sjögren, J.; Gohil, S.; Kenne, L.; Schnürer, J.; Broberg, A. Antifungal compounds from cultures of dairy propionibacteria type strains. FEMS Microbiol. Lett. 2007, 271, 310-315. [CrossRef] [PubMed]

78. Delavenne, E.; Ismail, R.; Pawtowski, A.; Mounier, J.; Barbier, G.; Le Blay, G. Assessment of lactobacilli strains as yogurt bioprotective cultures. Food Control 2013, 30, 206-213. [CrossRef] 
79. Garnier, L.; Leyva Salas, M.; Pinon, N.; Wiernasz, N.; Patowski, A.; Coton, E.; Mounier, J.; Valence, F. High-throughput method for antifungal activity screening in a cheese-mimicking model. 2017, manuscript in preparation.

80. Axel, C.; Röcker, B.; Brosnan, B.; Zannini, E.; Furey, A.; Coffey, A.; Arendt, E.K. Application of Lactobacillus amylovorus DSM19280 in gluten-free sourdough bread to improve the microbial shelf life. Food Microbiol. 2015, 47, 36-44. [CrossRef] [PubMed]

81. Bian, X.; Muhammad, Z.; Evivie, S.E.; Luo, G.-W.; Xu, M.; Huo, G.-C. Screening of antifungal potentials of Lactobacillus helveticus KLDS 1.8701 against spoilage microorganism and their effects on physicochemical properties and shelf life of fermented soybean milk during preservation. Food Control 2016, 66, 183-189. [CrossRef]

82. Nagaraja, H.; Chennappa, G.; Rakesh, S.; Naik, M.K.; Amaresh, Y.S.; Sreenivasa, M.Y. Antifungal activity of Azotobacter nigricans against trichothecene-producing Fusarium species associated with cereals. Food Sci. Biotechnol. 2016, 25, 1197-1204. [CrossRef]

83. Axel, C.; Brosnan, B.; Zannini, E.; Peyer, L.C.; Furey, A.; Coffey, A.; Arendt, E.K. Antifungal activities of three different Lactobacillus species and their production of antifungal carboxylic acids in wheat sourdough. Appl. Microbiol. Biotechnol. 2016, 100, 1701-1711. [CrossRef] [PubMed]

84. Kilani-Feki, O.; Khedher, S.B.; Dammak, M.; Kamoun, A.; Jabnoun-Khiareddine, H.; Daami-Remadi, M.; Tounsi, S. Improvement of antifungal metabolites production by Bacillus subtilis V26 for biocontrol of tomato postharvest disease. Biol. Control 2016, 95, 73-82. [CrossRef]

85. Li, W.; Zhang, H.; Li, P.; Apaliya, M.T.; Yang, Q.; Peng, Y.; Zhang, X. Biocontrol of postharvest green mold of oranges by Hanseniaspora uvarum Y3 in combination with phosphatidylcholine. Biol. Control 2016, 103, 30-38. [CrossRef]

86. Ma, H.; Sun, X.; Wang, M.; Gai, Y.; Chung, K.-R.; Li, H. The citrus postharvest pathogen Penicillium digitatum depends on the PdMpkB kinase for developmental and virulence functions. Int. J. Food Microbiol. 2016, 236, 167-176. [CrossRef] [PubMed]

87. Delavenne, E.; Cliquet, S.; Trunet, C.; Barbier, G.; Mounier, J.; Le Blay, G. Characterization of the antifungal activity of Lactobacillus harbinensis K.V9.3.1Np and Lactobacillus rhamnosus K.C8.3.1I in yogurt. Food Microbiol. 2015, 45, 10-17. [CrossRef] [PubMed]

88. Li, H.; Liu, L.; Zhang, S.; Uluko, H.; Cui, W.; Lv, J. Potential use of Lactobacillus casei AST18 as a bioprotective culture in yogurt. Food Control 2013, 34, 675-680. [CrossRef]

89. Lynch, K.M.; Pawlowska, A.M.; Brosnan, B.; Coffey, A.; Zannini, E.; Furey, A.; McSweeney, P.L.H.; Waters, D.M.; Arendt, E.K. Application of Lactobacillus amylovorus as an antifungal adjunct to extend the shelf-life of Cheddar cheese. Int. Dairy J. 2014, 34, 167-173. [CrossRef]

90. Axel, C.; Brosnan, B.; Zannini, E.; Furey, A.; Coffey, A.; Arendt, E.K. Antifungal sourdough lactic acid bacteria as biopreservation tool in quinoa and rice bread. Int. J. Food Microbiol. 2016. [CrossRef] [PubMed]

91. Bullerman, L.B.; Giesova, M.; Hassan, Y.; Deibert, D.; Ryu, D. Antifungal activity of sourdough bread cultures. In Advances in Food Mycology; Hocking, A.D., Pitt, J.I., Samson, R.A., Thrane, U., Eds.; Springer: Boston, MA, USA, 2006; Volume 571, pp. 307-316, ISBN 978-0-387-28385-2.

92. Coda, R.; Cassone, A.; Rizzello, C.G.; Nionelli, L.; Cardinali, G.; Gobbetti, M. Antifungal Activity of Wickerhamomyces anomalus and Lactobacillus plantarum during Sourdough Fermentation: Identification of Novel Compounds and Long-Term Effect during Storage of Wheat Bread. Appl. Environ. Microbiol. 2011, 77, 3484-3492. [CrossRef] [PubMed]

93. Corsetti, A.; Gobbetti, M.; Rossi, J.; Damiani, P. Antimould activity of sourdough lactic acid bacteria: identification of a mixture of organic acids produced by Lactobacillus sanfrancisco CB1. Appl. Microbiol. Biotechnol. 1998, 50, 253-256. [CrossRef] [PubMed]

94. Oliveira, P.; Brosnan, B.; Jacob, F.; Furey, A.; Coffey, A.; Zannini, E.; Arendt, E.K. Lactic acid bacteria bioprotection applied to the malting process. Part II: Substrate impact and mycotoxin reduction. Food Control 2015, 51, 444-452. [CrossRef]

95. Sangmanee, P.; Hongpattarakere, T. Inhibitory of multiple antifungal components produced by Lactobacillus plantarum K35 on growth, aflatoxin production and ultrastructure alterations of Aspergillus flavus and Aspergillus parasiticus. Food Control 2014, 40, 224-233. [CrossRef] 
96. Cray, J.A.; Bell, A.N.W.; Bhaganna, P.; Mswaka, A.Y.; Timson, D.J.; Hallsworth, J.E. The biology of habitat dominance; can microbes behave as weeds? The biology of habitat dominance. Microb. Biotechnol. 2013, 6, 453-492. [CrossRef] [PubMed]

97. Di Biase, M.; Lavermicocca, P.; Lonigro, S.L.; Valerio, F. Lactobacillus brevis-based bioingredient inhibits Aspergillus niger growth on pan bread. Ital. J. Agron. 2014, 9, 146. [CrossRef]

98. Ryu, E.H.; Yang, E.J.; Woo, E.R.; Chang, H.C. Purification and characterization of antifungal compounds from Lactobacillus plantarum HD1 isolated from kimchi. Food Microbiol. 2014, 41, 19-26. [CrossRef] [PubMed]

99. Gerez, C.L.; Fornaguera, M.J.; Obregozo, M.D.; Font de Valdez, G.; Torino, M.I. Antifungal starter culture for packed bread: Influence of two storage conditions. Rev. Argent. Microbiol. 2015, 47, 118-124. [CrossRef] [PubMed]

100. Saladino, F.; Luz, C.; Manyes, L.; Fernández-Franzón, M.; Meca, G. In vitro antifungal activity of lactic acid bacteria against mycotoxigenic fungi and their application in loaf bread shelf life improvement. Food Control 2016, 67, 273-277. [CrossRef]

101. Aunsbjerg, S.D.; Honoré, A.H.; Marcussen, J.; Ebrahimi, P.; Vogensen, F.K.; Benfeldt, C.; Skov, T.; Knøchel, S. Contribution of volatiles to the antifungal effect of Lactobacillus paracasei in defined medium and yogurt. Int. J. Food Microbiol. 2015, 194, 46-53. [CrossRef] [PubMed]

102. Gómez-Torres, N.; Ávila, M.; Delgado, D.; Garde, S. Effect of reuterin-producing Lactobacillus reuteri coupled with glycerol on the volatile fraction, odour and aroma of semi-hard ewe milk cheese. Int. J. Food Microbiol. 2016, 232, 103-110. [CrossRef] [PubMed]

103. Cheong, E.Y.L.; Sandhu, A.; Jayabalan, J.; Kieu Le, T.T.; Nhiep, N.T.; My Ho, H.T.; Zwielehner, J.; Bansal, N.; Turner, M.S. Isolation of lactic acid bacteria with antifungal activity against the common cheese spoilage mould Penicillium commune and their potential as biopreservatives in cheese. Food Control 2014, 46, 91-97. [CrossRef]

104. Fernandez, B.; Vimont, A.; Desfossés-Foucault, É.; Daga, M.; Arora, G.; Fliss, I. Antifungal activity of lactic and propionic acid bacteria and their potential as protective culture in cottage cheese. Food Control 2017, 78, 350-356. [CrossRef]

105. Axel, C.; Zannini, E.; Arendt, E.K.; Waters, D.M.; Czerny, M. Quantification of cyclic dipeptides from cultures of Lactobacillus brevis R2 $\Delta$ by HRGC/MS using stable isotope dilution assay. Anal. Bioanal. Chem. 2014, 406, 2433-2444. [CrossRef] [PubMed]

106. Peyer, L.C.; Zannini, E.; Arendt, E.K. Lactic acid bacteria as sensory biomodulators for fermented cereal-based beverages. Trends Food Sci. Technol. 2016, 54, 17-25. [CrossRef]

107. Peyer, L.C.; De Kruijf, M.; O’Mahony, J.; De Colli, L.; Danaher, M.; Zarnkow, M.; Jacob, F.; Arendt, E.K. Lactobacillus brevis R2 $\Delta$ as starter culture to improve biological and technological qualities of barley malt. Eur. Food Res. Technol. 2017. [CrossRef]

108. Ngang, J.-J.E.; Yadang, G.; Kamdem, S.L.S.; Kouebou, C.P.; Fanche, S.A.Y.; Kougan, D.L.T.; Tsoungui, A.; Etoa, F.-X. Antifungal properties of selected lactic acid bacteria and application in the biological control of ochratoxin A producing fungi during cocoa fermentation. Biocontrol Sci. Technol. 2015, 25, 245-259. [CrossRef]

109. Adedokun, E.O.; Rather, I.A.; Bajpai, V.K.; Park, Y.-H. Biocontrol efficacy of Lactobacillus fermentum YML014 against food spoilage moulds using the tomato puree model. Front. Life Sci. 2016, 9, 64-68. [CrossRef]

110. Varsha, K.K.; Devendra, L.; Shilpa, G.; Priya, S.; Pandey, A.; Nampoothiri, K.M. 2,4-Di-tert-butyl phenol as the antifungal, antioxidant bioactive purified from a newly isolated Lactococcus sp. Int. J. Food Microbiol. 2015, 211, 44-50. [CrossRef] [PubMed]

111. Gupta, R.; Srivastava, S. Antifungal effect of antimicrobial peptides (AMPs LR14) derived from Lactobacillus plantarum strain LR/14 and their applications in prevention of grain spoilage. Food Microbiol. 2014, 42, 1-7. [CrossRef] [PubMed]

112. Ahmad Rather, I.; Seo, B.J.; Rejish Kumar, V.J.; Choi, U.-H.; Choi, K.-H.; Lim, J.H.; Park, Y.-H. Isolation and characterization of a proteinaceous antifungal compound from Lactobacillus plantarum YML007 and its application as a food preservative. Lett. Appl. Microbiol. 2013, 57, 69-76. [CrossRef] [PubMed]

113. Gamba, R.R.; Caro, C.A.; Martínez, O.L.; Moretti, A.F.; Giannuzzi, L.; De Antoni, G.L.; León Peláez, A. Antifungal effect of kefir fermented milk and shelf life improvement of corn arepas. Int. J. Food Microbiol. 2016, 235, 85-92. [CrossRef] [PubMed]

114. Ghosh, R.; Barman, S.; Mukhopadhyay, A.; Mandal, N.C. Biological control of fruit-rot of jackfruit by rhizobacteria and food grade lactic acid bacteria. Biol. Control 2015, 83, 29-36. [CrossRef] 
115. Lipinska, L.; Klewicki, R.; Klewicka, E.; Kolodziejczyk, K.; Sojka, M.; Nowak, A. Antifungal Activity of Lactobacillus sp Bacteria in the Presence of Xylitol and Galactosyl-Xylitol. Biomed. Res. Int. 2016, 5897486. [CrossRef]

116. Matei, G.M.; Matei, S.; Matei, A.; Cornea, C.P.; Draghici, E.M.; Jerca, I.O. Bioprotection of fresh food products against blue mold using lactic acid bacteria with antifungal properties. Romanian Biotechnol. Lett. 2016, 21, 11201-11208.

117. Zhang, N.; Liu, J.; Li, J.; Chen, C.; Zhang, H.; Wang, H.-K.; Lu, F.-P. Characteristics and Application in Food Preservatives of Lactobacillus plantarum TK9 Isolated from Naturally Fermented Congee. Int. J. Food Eng. 2016, 12, 377-384. [CrossRef]

118. Kumar, S.N.; Sreekala, S.R.; Chandrasekaran, D.; Nambisan, B.; Anto, R.J. Biocontrol of Aspergillus Species on Peanut Kernels by Antifungal Diketopiperazine Producing Bacillus cereus Associated with Entomopathogenic Nematode. PLoS ONE 2014, 9, e106041. [CrossRef] [PubMed]

119. Gajbhiye, M.H.; Sathe, S.J.; Marathe, R.J.; Deshmukh, R.B. Antifungal Bacillus subtilis AFB22 from pomegranate with potential to control fruit rot. Res. J. Biotechnol. 2013, 8, 26-35.

120. Zhang, C.H.; Li, Y.; Liu, P.; Liu, M.J. Identification of two Bacillus amyloliquefaciens strains with high suppression to the key fruit pathogens of Chinese jujube. Biocontrol Sci. Technol. 2015, 25, 573-582. [CrossRef]

121. Kim, Y.S.; Balaraju, K.; Jeon, Y. Effects of rhizobacteria Paenibacillus polymyxa APEC136 and Bacillus subtilis APEC170 on biocontrol of postharvest pathogens of apple fruits. J. Zhejiang Univ. Sci. B 2016, 17, 931-940. [CrossRef] [PubMed]

122. Calvo, H.; Marco, P.; Blanco, D.; Oria, R.; Venturini, M.E. Potential of a new strain of Bacillus amyloliquefaciens BUZ-14 as a biocontrol agent of postharvest fruit diseases. Food Microbiol. 2017, 63, 101-110. [CrossRef] [PubMed]

123. Zhang, Z.; Chen, J.; Li, B.; He, C.; Chen, Y.; Tian, S. Influence of Oxidative Stress on Biocontrol Activity of Cryptococcus laurentii against Blue Mold on Peach Fruit. Front. Microbiol. 2017, 8. [CrossRef] [PubMed]

124. Gotor-Vila, A.; Teixidó, N.; Di Francesco, A.; Usall, J.; Ugolini, L.; Torres, R.; Mari, M. Antifungal effect of volatile organic compounds produced by Bacillus amyloliquefaciens CPA-8 against fruit pathogen decays of cherry. Food Microbiol. 2017, 64, 219-225. [CrossRef] [PubMed]

125. Passera, A.; Venturini, G.; Battelli, G.; Casati, P.; Penaca, F.; Quaglino, F.; Bianco, P.A. Competition assays revealed Paenibacillus pasadenensis strain R16 as a novel antifungal agent. Microbiol. Res. 2017, 198, 16-26. [CrossRef] [PubMed]

126. Wei, Y.; Mao, S.; Tu, K. Effect of preharvest spraying Cryptococcus laurentii on postharvest decay and quality of strawberry. Biol. Control 2014, 73, 68-74. [CrossRef]

127. Qin, X.; Xiao, H.; Xue, C.; Yu, Z.; Yang, R.; Cai, Z.; Si, L. Biocontrol of gray mold in grapes with the yeast Hanseniaspora uvarum alone and in combination with salicylic acid or sodium bicarbonate. Postharvest Biol. Technol. 2015, 100, 160-167. [CrossRef]

128. Campos-Martínez, A.; Velázquez-del Valle, M.G.; Flores-Moctezuma, H.E.; Suárez-Rodríguez, R.; Ramírez-Trujillo, J.A.; Hernández-Lauzardo, A.N. Antagonistic yeasts with potential to control Colletotrichum gloeosporioides (Penz.) Penz. \& Sacc. and Colletotrichum acutatum J.H. Simmonds on avocado fruits. Crop Prot. 2016, 89, 101-104. [CrossRef]

129. Zhou, Y.; Zhang, L.; Zeng, K. Efficacy of Pichia membranaefaciens combined with chitosan against Colletotrichum gloeosporioides in citrus fruits and possible modes of action. Biol. Control 2016, 96, 39-47. [CrossRef]

130. Ferraz, L.P.; da Cunha, T.; da Silva, A.C.; Kupper, K.C. Biocontrol ability and putative mode of action of yeasts against Geotrichum citri-aurantii in citrus fruit. Microbiol. Res. 2016, 188-189, 72-79. [CrossRef] [PubMed]

131. Perez, M.F.; Contreras, L.; Garnica, N.M.; Fernández-Zenoff, M.V.; Farías, M.E.; Sepulveda, M.; Ramallo, J.; Dib, J.R. Native Killer Yeasts as Biocontrol Agents of Postharvest Fungal Diseases in Lemons. PLoS ONE 2016, 11, e0165590. [CrossRef] [PubMed]

132. Grzegorczyk, M.; Żarowska, B.; Restuccia, C.; Cirvilleri, G. Postharvest biocontrol ability of killer yeasts against Monilinia fructigena and Monilinia fructicola on stone fruit. Food Microbiol. 2017, 61, 93-101. [CrossRef] [PubMed]

133. Zhimo, V.Y.; Dilip, D.; Sten, J.; Ravat, V.K.; Bhutia, D.D.; Panja, B.; Saha, J. Antagonistic Yeasts for Biocontrol of the Banana Postharvest Anthracnose Pathogen Colletotrichum musae. J. Phytopathol. 2017, 165, $35-43$. [CrossRef] 
134. Yang, Q.; Wang, H.; Zhang, H.; Zhang, X.; Apaliya, M.T.; Zheng, X.; Mahunu, G.K. Effect of Yarrowia lipolytica on postharvest decay of grapes caused by Talaromyces rugulosus and the protein expression profile of T. rugulosus. Postharvest Biol. Technol. 2017, 126, 15-22. [CrossRef]

135. Andrade, M.J.; Thorsen, L.; Rodríguez, A.; Córdoba, J.J.; Jespersen, L. Inhibition of ochratoxigenic moulds by Debaryomyces hansenii strains for biopreservation of dry-cured meat products. Int. J. Food Microbiol. 2014, 170, 70-77. [CrossRef] [PubMed]

136. Núñez, F.; Lara, M.S.; Peromingo, B.; Delgado, J.; Sánchez-Montero, L.; Andrade, M.J. Selection and evaluation of Debaryomyces hansenii isolates as potential bioprotective agents against toxigenic penicillia in dry-fermented sausages. Food Microbiol. 2015, 46, 114-120. [CrossRef] [PubMed]

137. Bernáldez, V.; Córdoba, J.J.; Rodríguez, M.; Cordero, M.; Polo, L.; Rodríguez, A. Effect of Penicillium nalgiovense as protective culture in processing of dry-fermented sausage "salchichón". Food Control 2013, 32, 69-76. [CrossRef]

138. Alía, A.; Andrade, M.J.; Rodríguez, A.; Reyes-Prieto, M.; Bernáldez, V.; Córdoba, J.J. Identification and control of moulds responsible for black spot spoilage in dry-cured ham. Meat Sci. 2016, 122, 16-24. [CrossRef] [PubMed]

139. Gong, C.; Liu, Y.; Liu, S.; Cheng, M.; Zhang, Y.; Wang, R.; Chen, H.; Li, J.; Chen, X.; Wang, A. Analysis of Clonostachys rosea-induced resistance to grey mould disease and identification of the key proteins induced in tomato fruit. Postharvest Biol. Technol. 2017, 123, 83-93. [CrossRef]

140. Schnürer, J.; Magnusson, J. Antifungal lactic acid bacteria as biopreservatives. Trends Food Sci. Technol. 2005, 16, 70-78. [CrossRef]

141. Scholz, C.F.P.; Kilian, M. The natural history of cutaneous propionibacteria, and reclassification of selected species within the genus Propionibacterium to the proposed novel genera Acidipropionibacterium gen. nov., Cutibacterium gen. nov. and Pseudopropionibacterium gen. nov. Int. J. Syst. Evol. Microbiol. 2016, 66, 4422-4432. [CrossRef] [PubMed]

142. Crowley, S.; Mahony, J.; van Sinderen, D. Comparative analysis of two antifungal Lactobacillus plantarum isolates and their application as bioprotectants in refrigerated foods. J. Appl. Microbiol. 2012, 113, 1417-1427. [CrossRef] [PubMed]

143. Dal Bello, F.; Clarke, C.I.; Ryan, L.A.M.; Ulmer, H.; Schober, T.J.; Ström, K.; Sjögren, J.; van Sinderen, D.; Schnürer, J.; Arendt, E.K. Improvement of the quality and shelf life of wheat bread by fermentation with the antifungal strain Lactobacillus plantarum FST 1.7. J. Cereal Sci. 2007, 45, 309-318. [CrossRef]

144. Zhang, X.; Zhang, S.; Shi, Y.; Shen, F.; Wang, H. A new high phenyl lactic acid-yielding Lactobacillus plantarum IMAU10124 and a comparative analysis of lactate dehydrogenase gene. FEMS Microbiol. Lett. 2014, 356, 89-96. [CrossRef] [PubMed]

145. Muhialdin, B.J.; Hassan, Z.; Bakar, F.A.; Saari, N. Identification of antifungal peptides produced by Lactobacillus plantarum IS10 grown in the MRS broth. Food Control 2016, 59, 27-30. [CrossRef]

146. Sagdic, O.; Ozturk, I.; Yapar, N.; Yetim, H. Diversity and probiotic potentials of lactic acid bacteria isolated from gilaburu, a traditional Turkish fermented European cranberrybush (Viburnum opulus L.) fruit drink. Food Res. Int. 2014, 64, 537-545. [CrossRef]

147. Garofalo, C.; Zannini, E.; Aquilanti, L.; Silvestri, G.; Fierro, O.; Picariello, G.; Clementi, F. Selection of Sourdough Lactobacilli with Antifungal Activity for Use as Biopreservatives in Bakery Products. J. Agric. Food Chem. 2012, 60, 7719-7728. [CrossRef] [PubMed]

148. Arendt, E.K.; Dal Bello, F.; Ryan, L. Increasing the Shelf Life of Bakery and Patisserie Products by Using the Antifungal Lactobacillus Amylovorus DSM 19280. US Patent WO200,9141,427 A2, 26 November 2009.

149. Hemme, D.; Foucaud-Scheunemann, C. Leuconostoc, characteristics, use in dairy technology and prospects in functional foods. Int. Dairy J. 2004, 14, 467-494. [CrossRef]

150. Kalschne, D.L.; Womer, R.; Mattana, A.; Sarmento, C.M.P.; Colla, L.M.; Colla, E. Characterization of the spoilage lactic acid bacteria in in "sliced vacuum-packed cooked ham". Braz. J. Microbiol. 2015, 46, 173-181. [CrossRef] [PubMed]

151. Sadeghi, A.; Raeisi, M.; Ebrahimi, M.; Sadeghi, B. Antifungal Activity of Pediococcus pentosaceus Isolated from Whole Barley Sourdough. J. Food Qual. Hazards Control 2016, 3, 30-36.

152. Oliveira, P.M.; Brosnan, B.; Furey, A.; Coffey, A.; Zannini, E.; Arendt, E.K. Lactic acid bacteria bioprotection applied to the malting process. Part I: Strain characterization and identification of antifungal compounds. Food Control 2015, 51, 433-443. [CrossRef] 
153. Stackebrandt, E.; Cummins, C.S.; Johnson, J.L. Family Propionibacteriaceae: The genus Propionibacterium. In The Prokaryotes; Dworkin, M., Falkow, S., Rosenberg, E., Schleifer, K.-H., Stackebrandt, E., Eds.; Springer: New York, NY, USA, 2006; pp. 400-418, ISBN 978-0-387-25493-7.

154. Falardeau, J.; Wise, C.; Novitsky, L.; Avis, T.J. Ecological and Mechanistic Insights into the Direct and Indirect Antimicrobial Properties of Bacillus subtilis Lipopeptides on Plant Pathogens. J. Chem. Ecol. 2013, 39, 869-878. [CrossRef] [PubMed]

155. Pretorius, D.; van Rooyen, J.; Clarke, K.G. Enhanced production of antifungal lipopeptides by Bacillus amyloliquefaciens for biocontrol of postharvest disease. New Biotechnol. 2015, 32, 243-252. [CrossRef] [PubMed]

156. Li, X.; Zhang, Y.; Wei, Z.; Guan, Z.; Cai, Y.; Liao, X. Antifungal Activity of Isolated Bacillus amyloliquefaciens SYBC H47 for the Biocontrol of Peach Gummosis. PLoS ONE 2016, 11, e0162125. [CrossRef] [PubMed]

157. Muccilli, S.; Restuccia, C. Bioprotective Role of Yeasts. Microorganisms 2015, 3, 588-611. [CrossRef] [PubMed]

158. Hara, S.; Iimura, Y.; Otsuka, K. Breeding of Useful Killer Wine Yeasts. Am. J. Enol. Vitic. 1980, 31, $28-33$.

159. Janisiewicz, W.J.; Korsten, L. Biological cotrol of postharvest diseases of fruits. Annu. Rev. Phytopathol. 2002, 40, 411-441. [CrossRef] [PubMed]

160. Hatoum, R.; Labrie, S.; Fliss, I. Antimicrobial and Probiotic Properties of Yeasts: From Fundamental to Novel Applications. Front. Microbiol. 2012, 3. [CrossRef] [PubMed]

161. Chanchaichaovivat, A.; Panijpan, B.; Ruenwongsa, P. Putative modes of action of Pichia guilliermondii strain R13 in controlling chilli anthracnose after harvest. Biol. Control 2008, 47, 207-215. [CrossRef]

162. Zhang, X.-Y.; Zhang, Y.; Xu, X.-Y.; Qi, S.-H. Diverse Deep-Sea Fungi from the South China Sea and Their Antimicrobial Activity. Curr. Microbiol. 2013, 67, 525-530. [CrossRef] [PubMed]

163. Wang, Y.; Yu, T.; Li, Y.; Cai, D.; Liu, X.; Lu, H.; Zheng, X. Postharvest biocontrol of Alternaria alternata in Chinese winter jujube by Rhodosporidium paludigenum. J. Appl. Microbiol. 2009, 107, 1492-1498. [CrossRef] [PubMed]

164. Banjara, N.; Suhr, M.J.; Hallen-Adams, H.E. Diversity of Yeast and Mold Species from a Variety of Cheese Types. Curr. Microbiol. 2015, 70, 792-800. [CrossRef] [PubMed]

165. Hammes, W.P.; Knauf, H.J. Starters in the processing of meat products. Meat Sci. 1994, 36, 155-168. [CrossRef]

166. Delgado, J.; Acosta, R.; Rodríguez-Martín, A.; Bermúdez, E.; Núñez, F.; Asensio, M.A. Growth inhibition and stability of PgAFP from Penicillium chrysogenum against fungi common on dry-ripened meat products. Int. J. Food Microbiol. 2015, 205, 23-29. [CrossRef] [PubMed]

167. Delgado, J.; Owens, R.A.; Doyle, S.; Asensio, M.A.; Núñez, F. Impact of the antifungal protein PgAFP from Penicillium chrysogenum on the protein profile in Aspergillus flavus. Appl. Microbiol. Biotechnol. 2015, 99, 8701-8715. [CrossRef] [PubMed]

168. Cray, J.A.; Bhaganna, P.; Singhal, R.S.; Patil, S.V.; Saha, D.; Chakraborty, R; Timson, D.J.; Hallsworth, J.E. Modern fungicides and antifungal compounds VII. In Proceedings of the 17th International Reinhardsbrunn Symposium, April 21-25, 2013, Friedrichroda, Germany; DPG Spectrum Phytomedizin; DPG-Verl: Braunschweig, Germany, 2014; ISBN 978-3-941261-13-6.

169. Thierry, A.; Deutsch, S.-M.; Falentin, H.; Dalmasso, M.; Cousin, F.J.; Jan, G. New insights into physiology and metabolism of Propionibacterium freudenreichii. Int. J. Food Microbiol. 2011, 149, 19-27. [CrossRef] [PubMed]

170. Dagnas, S.; Gauvry, E.; Onno, B.; Membré, J.-M. Quantifying Effect of Lactic, Acetic, and Propionic Acids on Growth of Molds Isolated from Spoiled Bakery Products. J. Food Prot. 2015, 78, 1689-1698. [CrossRef] [PubMed]

171. Axelsson, L.T.; Chung, T.C.; Dobrogosz, W.J.; Lindgren, S.E. Production of a Broad Spectrum Antimicrobial Substance by Lactobacillus reuteri. Microb. Ecol. Health Dis. 1989, 2, 131-136. [CrossRef]

172. Black, B.A.; Zannini, E.; Curtis, J.M.; Ganzle, M.G. Antifungal Hydroxy Fatty Acids Produced during Sourdough Fermentation: Microbial and Enzymatic Pathways, and Antifungal Activity in Bread. Appl. Environ. Microbiol. 2013, 79, 1866-1873. [CrossRef] [PubMed]

173. Broberg, A.; Jacobsson, K.; Strom, K.; Schnurer, J. Metabolite Profiles of Lactic Acid Bacteria in Grass Silage. Appl. Environ. Microbiol. 2007, 73, 5547-5552. [CrossRef] [PubMed]

174. Li, H.; Liu, L.; Zhang, S.; Cui, W.; Lv, J. Identification of Antifungal Compounds Produced by Lactobacillus casei AST18. Curr. Microbiol. 2012, 65, 156-161. [CrossRef] [PubMed] 
175. Mieszkin, S.; Hymery, N.; Debaets, S.; Coton, E.; Le Blay, G.; Valence, F.; Mounier, J. Action mechanisms involved in the bioprotective effect of Lactobacillus harbinensis K.V9.3.1.Np against Yarrowia lipolytica in fermented milk. Int. J. Food Microbiol. 2017. [CrossRef] [PubMed]

176. Strom, K.; Sjogren, J.; Broberg, A.; Schnurer, J. Lactobacillus plantarum MiLAB 393 Produces the Antifungal Cyclic Dipeptides Cyclo(L-Phe-L-Pro) and Cyclo(L-Phe-trans-4-OH-L-Pro) and 3-Phenyllactic Acid. Appl. Environ. Microbiol. 2002, 68, 4322-4327. [CrossRef] [PubMed]

177. Valerio, F.; Di Biase, M.; Lattanzio, V.M.T.; Lavermicocca, P. Improvement of the antifungal activity of lactic acid bacteria by addition to the growth medium of phenylpyruvic acid, a precursor of phenyllactic acid. Int. J. Food Microbiol. 2016, 222, 1-7. [CrossRef] [PubMed]

178. Yang, E.J.; Chang, H.C. Purification of a new antifungal compound produced by Lactobacillus plantarum AF1 isolated from kimchi. Int. J. Food Microbiol. 2010, 139, 56-63. [CrossRef] [PubMed]

179. Le Lay, C.; Coton, E.; Le Blay, G.; Chobert, J.-M.; Haertlé, T.; Choiset, Y.; Van Long, N.N.; Meslet-Cladière, L.; Mounier, J. Identification and quantification of antifungal compounds produced by lactic acid bacteria and propionibacteria. Int. J. Food Microbiol. 2016. [CrossRef] [PubMed]

180. Paik, H.D.; Glatz, B.A. Purification and partial amino acid sequence of propionicin PLG-1, a bacteriocin produced by Propionibacterium thoenii P127. Le Lait 1995, 75, 367-377. [CrossRef]

181. Schwenninger, S.M.; Lacroix, C.; Truttmann, S.; Jans, C.; Spörndli, C.; Bigler, L.; Meile, L. Characterization of low-molecular-weight antiyeast metabolites produced by a food-protective Lactobacillus-Propionibacterium coculture. J. Food Prot. 2008, 71, 2481-2487. [CrossRef] [PubMed]

182. Theron, M.M.; Lues, J.F.R. Organic Acids and Food Preservation; CRC Press, Taylor \& Francis Group: Boca Raton, FL, USA, 2011; ISBN 978-1-4200-7842-8.

183. Stratford, M.; Nebe-von-Caron, G.; Steels, H.; Novodvorska, M.; Ueckert, J.; Archer, D.B. Weak-acid preservatives: $\mathrm{pH}$ and proton movements in the yeast Saccharomyces cerevisiae. Int. J. Food Microbiol. 2013, 161, 164-171. [CrossRef] [PubMed]

184. Cray, J.A.; Russell, J.T.; Timson, D.J.; Singhal, R.S.; Hallsworth, J.E. A universal measure of chaotropicity and kosmotropicity: A universal measure of chao- and kosmotropicity. Environ. Microbiol. 2013, 15, 287-296. [CrossRef] [PubMed]

185. Cray, J.A.; Stevenson, A.; Ball, P.; Bankar, S.B.; Eleutherio, E.C.; Ezeji, T.C.; Singhal, R.S.; Thevelein, J.M.; Timson, D.J.; Hallsworth, J.E. Chaotropicity: A key factor in product tolerance of biofuel-producing microorganisms. Curr. Opin. Biotechnol. 2015, 33, 228-259. [CrossRef] [PubMed]

186. Brosnan, B.; Coffey, A.; Arendt, E.K.; Furey, A. Rapid identification, by use of the LTQ Orbitrap hybrid FT mass spectrometer, of antifungal compounds produced by lactic acid bacteria. Anal. Bioanal. Chem. 2012, 403, 2983-2995. [CrossRef] [PubMed]

187. Brosnan, B.; Coffey, A.; Arendt, E.K.; Furey, A. The QuEChERS approach in a novel application for the identification of antifungal compounds produced by lactic acid bacteria cultures. Talanta 2014, 129, 364-373. [CrossRef] [PubMed]

188. Brosnan, B.; Coffey, A.; Arendt, E.K.; Furey, A. A comprehensive investigation into sample extraction and method validation for the identification of antifungal compounds produced by lactic acid bacteria using HPLC-UV/DAD. Anal. Methods 2014, 6, 5331-5344. [CrossRef]

189. Crowley, S.; Mahony, J.; Morrissey, J.P.; van Sinderen, D. Transcriptomic and morphological profiling of Aspergillus fumigatus Af293 in response to antifungal activity produced by Lactobacillus plantarum 16. Microbiology 2013, 159, 2014-2024. [CrossRef] [PubMed]

190. Coda, R.; Rizzello, C.G.; Di Cagno, R.; Trani, A.; Cardinali, G.; Gobbetti, M. Antifungal activity of Meyerozyma guilliermondii: Identification of active compounds synthesized during dough fermentation and their effect on long-term storage of wheat bread. Food Microbiol. 2013, 33, 243-251. [CrossRef] [PubMed]

191. Delgado, J.; Peromingo, B.; Núñez, F.; Asensio, M.A. Use of molds and their antifungal proteins for biocontrol of toxigenic molds on dry-ripened cheese and meats. Curr. Opin. Food Sci. 2016, 11, 40-45. [CrossRef]

192. Haidar, R.; Fermaud, M.; Calvo-Garrido, C.; Roudet, J.; Deschamps, A. Modes of action for biological control of Botrytis cinerea by antagonistic bacteria. Phytopathol. Mediterr. 2016, 55, 301-322. [CrossRef]

193. Ongena, M.; Jacques, P. Bacillus lipopeptides: Versatile weapons for plant disease biocontrol. Trends Microbiol. 2008, 16, 115-125. [CrossRef] [PubMed]

194. Rautenbach, M.; Troskie, A.M.; Vosloo, J.A. Antifungal peptides: To be or not to be membrane active. Biochimie 2016, 130, 132-145. [CrossRef] [PubMed] 
195. Selitrennikoff, C.P. Antifungal Proteins. Appl. Environ. Microbiol. 2001, 67, 2883-2894. [CrossRef] [PubMed]

196. Sumi, C.D.; Yang, B.W.; Yeo, I.-C.; Hahm, Y.T. Antimicrobial peptides of the genus Bacillus: A new era for antibiotics. Can. J. Microbiol. 2015, 61, 93-103. [CrossRef] [PubMed]

197. Tan, Z.; Lin, B.; Zhang, R. A novel antifungal protein of Bacillus subtilis B25. SpringerPlus 2013, 2, 543. [CrossRef] [PubMed]

198. Di Francesco, A.; Martini, C.; Mari, M. Biological control of postharvest diseases by microbial antagonists: How many mechanisms of action? Eur. J. Plant Pathol. 2016, 145, 711-717. [CrossRef]

199. Rouissi, W.; Ugolini, L.; Martini, C.; Lazzeri, L.; Mari, M. Control of Postharvest Fungal Pathogens by Antifungal Compounds from Penicillium expansum. J. Food Prot. 2013, 76, 1879-1886. [CrossRef] [PubMed]

200. Li, H.; Zhang, S.; Lu, J.; Liu, L.; Uluko, H.; Pang, X.; Sun, Y.; Xue, H.; Zhao, L.; Kong, F.; et al. Antifungal activities and effect of Lactobacillus casei AST18 on the mycelia morphology and ultrastructure of Penicillium chrysogenum. Food Control 2014, 43, 57-64. [CrossRef]

201. Nally, M.C.; Pesce, V.M.; Maturano, Y.P.; Rodriguez Assaf, L.A.; Toro, M.E.; Castellanos de Figueroa, L.I.; Vazquez, F. Antifungal modes of action of Saccharomyces and other biocontrol yeasts against fungi isolated from sour and grey rots. Int. J. Food Microbiol. 2015, 204, 91-100. [CrossRef] [PubMed]

202. Hua, S.S.T.; Beck, J.J.; Sarreal, S.B.L.; Gee, W. The major volatile compound 2-phenylethanol from the biocontrol yeast, Pichia anomala, inhibits growth and expression of aflatoxin biosynthetic genes of Aspergillus flavus. Mycotoxin Res. 2014, 30, 71-78. [CrossRef] [PubMed]

203. Rahman, M. Bacillus spp.: A promising biocontrol agent of root, foliar, and postharvest diseases of plants. In Bacilli and Agrobiotechnology; Islam, M.T., Rahman, M., Pandey, P., Jha, C.K., Aeron, A., Eds.; Springer International Publishing: Cham, Switzerland, 2016; pp. 113-141, ISBN 978-3-319-44408-6.

204. Li, C.; Wang, J.; Luo, C.; Ding, W.; Cox, D.G. A new cyclopeptide with antifungal activity from the co-culture broth of two marine mangrove fungi. Nat. Prod. Res. 2014, 28, 616-621. [CrossRef] [PubMed]

205. Klewicka, E. Antifungal activity of lactic acid bacteria of genus Lactobacillus sp in the presence of polyols. Acta Aliment. 2007, 36, 495-499. [CrossRef]

206. Serrazanetti, D.I.; Guerzoni, M.E.; Corsetti, A.; Vogel, R. Metabolic impact and potential exploitation of the stress reactions in lactobacilli. Food Microbiol. 2009, 26, 700-711. [CrossRef] [PubMed]

207. Qin, G.Z.; Tian, S.P.; Xu, Y.; Wan, Y.K. Enhancement of biocontrol efficacy of antagonistic yeasts by salicylic acid in sweet cherry fruit. Physiol. Mol. Plant Pathol. 2003, 62, 147-154. [CrossRef]

208. Geremew, T.; Kebede, A.; Andualem, B. The role of spices and lactic acid bacteria as antimicrobial agent to extend the shelf life of metata ayib (traditional Ethiopian spiced fermented cottage cheese). J. Food Sci. Technol. 2015, 52, 5661-5670. [CrossRef] [PubMed]

209. EFSA Panel on Biological Hazards (BIOHAZ). Update of the list of QPS-recommended biological agents intentionally added to food or feed as notified to EFSA 4: Suitability of taxonomic units notified to EFSA until March 2016. EFSA J. 2016, 14. [CrossRef]

210. Bourdichon, F.; Casaregola, S.; Farrokh, C.; Frisvad, J.C.; Gerds, M.L.; Hammes, W.P.; Harnett, J.; Huys, G.; Laulund, S.; Ouwehand, A.; et al. Food fermentations: Microorganisms with technological beneficial use. Int. J. Food Microbiol. 2012, 154, 87-97. [CrossRef] [PubMed]

211. FDA Generally Recognized as Safe (GRAS). Available online: https://www.fda.gov/Food/ IngredientsPackagingLabeling/GRAS/ (accessed on 9 April 2017).

212. Coton, M.; Lebreton, M.; Leyva Salas, M.; Navarro, M.; Patowsky, A.; Le Blay, G.; Valence, F.; Coton, E.; Mounier, J. Safety risk assessment of potential antifungal lactic acid bacteria and propionibacteria. 2017, in preparation.

213. Gasser, F. Safety of lactic acid bacteria and their occurrence in human clinical infections. Bull. Inst. Pasteur 1994, 92, 45-67.

214. Mogensen, G.; Salminen, S.; O’brien, J.; Ouwehand, A.; Holzapfel, W.; Shortt, C.; Fonden, R.; Miller, G.; Donohue, D.; Playne, M. Food microorganisms: Health benefits, safety evaluation and strains with documented history of use in foods. Bull. Int. Dairy Fed. 2002, 377, 4-9.

215. Vankerckhoven, V.; Huys, G.; Vancanneyt, M.; Vael, C.; Klare, I.; Romond, M.-B.; Entenza, J.M.; Moreillon, P.; Wind, R.D.; Knol, J.; et al. Biosafety assessment of probiotics used for human consumption: Recommendations from the EU-PROSAFE project. Trends Food Sci. Technol. 2008, 19, 102-114. [CrossRef] 
216. Ladero, V.; Fernández, M.; Calles-Enríquez, M.; Sánchez-Llana, E.; Cañedo, E.; Martín, M.C.; Alvarez, M.A. Is the production of the biogenic amines tyramine and putrescine a species-level trait in enterococci? Food Microbiol. 2012, 30, 132-138. [CrossRef] [PubMed]

217. Martín, J.F.; Coton, M. Blue cheese. In Fermented Foods in Health and Disease Prevention; Elsevier: Amsterdam, The Netherlands, 2017; pp. 275-303, ISBN 978-0-12-802309-9.

218. Spano, G.; Russo, P.; Lonvaud-Funel, A.; Lucas, P.; Alexandre, H.; Grandvalet, C.; Coton, E.; Coton, M.; Barnavon, L.; Bach, B.; et al. Biogenic amines in fermented foods. Eur. J. Clin. Nutr. 2010, 64, S95-S100. [CrossRef] [PubMed]

219. Coton, E.; Coton, M. Evidence of horizontal transfer as origin of strain to strain variation of the tyramine production trait in Lactobacillus brevis. Food Microbiol. 2009, 26, 52-57. [CrossRef] [PubMed]

220. Marcobal, Á.; Martín-Álvarez, P.J.; Polo, M.C.; Muñoz, R.; Moreno-Arribas, M.V. Formation of biogenic amines throughout the industrial manufacture of red wine. J. Food Prot. 2006, 69, 397-404. [CrossRef] [PubMed]

221. Ladero, V.; Calles-Enríquez, M.; Fernández, M.; Alvarez, A.M. Toxicological effects of dietary biogenic amines. Curr. Nutr. Food Sci. 2010, 6, 145-156. [CrossRef]

222. Sillasantos, M.H. Biogenic amines: Their importance in foods. Int. J. Food Microbiol. 1996, 29, 213-231. [CrossRef]

223. Melin, P.; Hakansson, S.; Eberhard, T.H.; Schnurer, J. Survival of the biocontrol yeast Pichia anomala after long-term storage in liquid formulations at different temperatures, assessed by flow cytometry. J. Appl. Microbiol. 2006, 100, 264-271. [CrossRef] [PubMed]

224. Melin, P.; Sundh, I.; Hakansson, S.; Schnurer, J. Biological preservation of plant derived animal feed with antifungal microorganisms: Safety and formulation aspects. Biotechnol. Lett. 2007, 29, 1147-1154. [CrossRef] [PubMed]

225. Li, B.Q.; Tian, S.P. Effects of trehalose on stress tolerance and biocontrol efficacy of Cryptococcus laurentii. J. Appl. Microbiol. 2006, 100, 854-861. [CrossRef] [PubMed]

226. Gálvez, A.; Abriouel, H.; López, R.L.; Omar, N.B. Bacteriocin-based strategies for food biopreservation. Int. J. Food Microbiol. 2007, 120, 51-70. [CrossRef] [PubMed]

227. Pfeiler, E.A.; Klaenhammer, T.R. The genomics of lactic acid bacteria. Trends Microbiol. 2007, 15, 546-553. [CrossRef] [PubMed]

228. Rouse, S.; Harnett, D.; Vaughan, A.; Van Sinderen, D. Lactic acid bacteria with potential to eliminate fungal spoilage in foods. J. Appl. Microbiol. 2008, 104, 915-923. [CrossRef] [PubMed]

229. Schillinger, U.; Villarreal, J.V. Inhibition of Penicillium nordicum in MRS medium by lactic acid bacteria isolated from foods. Food Control 2010, 21, 107-111. [CrossRef]

230. Zhang, Y.-Q.; Gamarra, S.; Garcia-Effron, G.; Park, S.; Perlin, D.S.; Rao, R. Requirement for Ergosterol in V-ATPase Function Underlies Antifungal Activity of Azole Drugs. PLoS Pathog. 2010, 6, e1000939. [CrossRef] [PubMed]

(C) 2017 by the authors. Licensee MDPI, Basel, Switzerland. This article is an open access article distributed under the terms and conditions of the Creative Commons Attribution (CC BY) license (http://creativecommons.org/licenses/by/4.0/). 